\title{
Developments of some new results that weaken certain conditions of fractional type differential equations
}

\author{
Shahid Bashir ${ }^{1}$, Naeem Saleem², Hassen Aydi ${ }^{3,4,5^{*}}$ (D), Syed Muhammad Husnine ${ }^{1}$ and Asma Al Rwaily ${ }^{6}$
}

\author{
"Correspondence: \\ hassen.aydi@isima.rnu.tn \\ ${ }^{3}$ Institut Supérieur d'Informatique et \\ des Techniques de Communication, \\ Université de Sousse, Sousse, Tunisia \\ ${ }^{4}$ China Medical University Hospital, \\ China Medical University, Taichung, \\ 40402, Taiwan \\ Full list of author information is \\ available at the end of the article
}

\begin{abstract}
We introduce double and triple F-expanding mappings. We prove related fixed point theorems. Based on our obtained results, we also prove the existence of a solution for fractional type differential equations by using a weaker condition than the sufficient small Lipschitz constant studied by Mehmood and Ahmad (AIMS Math. 5:385-398, 2019) and Hanadi et al. (Mathematics 8:1168, 2020). As applications, we ensure the existence of a unique solution of a boundary value problem for a second-order differential equation.
\end{abstract}

MSC: $47 \mathrm{H} 10 ; 47 \mathrm{H} 19 ; 54 \mathrm{H} 25$

\section{Introduction}

In 2012, Wardowski [3] generalized the Banach contraction principle by introducing a new type of contractions, called $F$-contractions, and established a unique related fixed point theorem. This modification of BCP motivated many researchers to study further possibilities of its extensions [4-25]. In 2017, Gornicki [26] presented some new fixed point results for $F$-expanding mappings. We modify this setting by introducing multiple $F$ functions. The usage of multiple $F$ functions permits to find solutions for an extensive range of integral equations.

The nonlinear fractional differential equations have a valuable role in various fields of science, such as engineering, biology, fluid mechanics, physics, chemistry, bio-physics. For more details, see [21, 22, 27-37]. After establishing the fixed point theorems for expanding type mappings, we provide some new sufficient conditions for the existence of solutions of an integral boundary value problem for a scalar nonlinear Caputo fractional differential equation with fractional order in $(1,2)$. We also compare the obtained result with known ones in the literature. Furthermore, we use our obtained results to find a solution of an engineering problem, in which the transformed mathematical model of a problem representing activation of a spring affected by an external force is a boundary value problem for a second-order differential equation.

(c) The Author(s) 2021. This article is licensed under a Creative Commons Attribution 4.0 International License, which permits use sharing, adaptation, distribution and reproduction in any medium or format, as long as you give appropriate credit to the original author(s) and the source, provide a link to the Creative Commons licence, and indicate if changes were made. The images or other third party material in this article are included in the article's Creative Commons licence, unless indicated otherwise in a credit line to the material. If material is not included in the article's Creative Commons licence and your intended use is not permitted by statutory regulation or exceeds the permitted use, you will need to obtain permission directly from the copyright holder. To view a copy of this licence, visit http://creativecommons.org/licenses/by/4.0/. 


\section{Preliminaries}

In this paper, $\mathbb{N}, \mathbb{N}_{0}, \mathbb{R}$, and $\mathbb{R}_{+}$denote the set of natural numbers, $\mathbb{N} \cup\{0\}$, real numbers, and positive real numbers, respectively. Throughout the paper, every set $X$ taken into account is nonempty. Wardowski [3] defined the concept of $F$-contractions as follows.

Definition 2.1 ([3]) Let $(X, \mathcal{D})$ be a metric space. A mapping $T: X \mapsto X$ is said to be an $F$-contraction if there is a real number $\tau>0$ such that, for all $x, y \in X$,

$$
[\mathcal{D}(T x, T y)>0 \text { implies } \tau+F(\mathcal{D}(T x, T y)) \leq F(\mathcal{D}(x, y))]
$$

where $F: \mathbb{R}_{+}=(0, \infty) \mapsto \mathbb{R}$ is a function satisfying the following conditions:

(F1) $F$ is strictly increasing, that is, for all $x, y \in \mathbb{R}_{+}, x<y, F(x)<F(y)$;

(F2) For each sequence $\left\{\alpha_{n}\right\}_{n=1}^{\infty}$ of positive numbers, $\lim _{n \rightarrow \infty} \alpha_{n}=0$ if and only if $\lim _{n \rightarrow \infty} F\left(\alpha_{n}\right)=-\infty$;

(F3) There is $k \in(0,1)$ such that $\lim _{\alpha \mapsto 0^{+}} \alpha^{k} F(\alpha)=0$.

Denote by $\mathcal{F}$ the set of all functions satisfying conditions (F1)-(F3).

Example 2.1 Let $F_{i}: \mathbb{R}_{+} \mapsto \mathbb{R}(i=1,2,3,4)$ be defined by

(i) $F_{1}(t)=\ln t$.

(ii) $F_{2}(t)=t+\ln t$.

(iii) $F_{3}(t)=-\frac{1}{\sqrt{t}}$.

(iv) $F_{4}(t)=\ln \left(t^{2}+t\right)$.

Then $F_{1}, F_{2}, F_{3}, F_{4} \in \mathcal{F}$.

Remark 2.1 From the conditions of $F$-contractions, it is easy to conclude that every $F$ contraction mapping is necessarily continuous.

Further, Wardowski [3] stated a modified version of the Banach contraction principle as follows.

Theorem 2.1 ([3]) Let $(X, \mathcal{D})$ be a complete metric space and $T: X \mapsto X$ be an Fcontraction. Then $T$ has a unique fixed point, say $x^{*} \in X$, and for every $x \in X$, the sequence $\left\{T^{n} x\right\}_{n \in N}$ converges to $x^{*}$.

For details on $F$-contraction mappings, see $[8,9,38,39]$. The concept of $F$-expanding mappings is given as follows.

Definition 2.2 ([26]) Let $(X, \mathcal{D})$ be a metric space. A mapping $T: X \mapsto X$ is said to be $F$-expanding if there are $F \in \mathcal{F}$ and a real number $\tau>0$ such that, for all $x, y \in X$,

$$
[\mathcal{D}(T x, T y)>0 \text { implies } F(\mathcal{D}(T x, T y)) \geq F(\mathcal{D}(x, y))+\tau]
$$

\section{Main results}

Firstly, we introduce two types of double $F$-expanding mappings that generalized $F$ expanding mappings. 
Definition 3.1 Let $(X, \mathcal{D})$ be a metric space. A mapping $T: X \mapsto X$ is said to be double $F$-expanding of type I, if there exist a real number $\tau>0$ and $F_{1}, F_{2} \in \mathcal{F}$ such that, for all $x, y \in X$, we have

$$
\begin{aligned}
\mathcal{D}\left(T^{2} x, T^{2} y\right)>0 \quad \text { and } \quad \mathcal{D}(T x, T y)>0 \\
\text { imply } \min \left\{F_{2}\left(\mathcal{D}\left(T^{2} x, T^{2} y\right)\right), F_{1}(\mathcal{D}(T x, T y))\right\} \\
\geq \alpha_{2} F_{2}(\mathcal{D}(x, y))+\alpha_{1} F_{1}(\mathcal{D}(x, y))+\tau,
\end{aligned}
$$

where

$$
\left\{\begin{array}{c}
\alpha_{1}=0, \alpha_{2}=1, \text { if } F_{2}\left(\mathcal{D}\left(T^{2} x, T^{2} y\right)\right) \leq F_{1}(\mathcal{D}(T x, T y)) \\
\alpha_{1}=1, \alpha_{2}=0, \text { if } F_{2}\left(\mathcal{D}\left(T^{2} x, T^{2} y\right)\right)>F_{1}(\mathcal{D}(T x, T y))
\end{array}\right\} .
$$

Remark 3.1 For some $x, y \in X$, the conditions of Definition 3.1 yield either $F_{2}\left(\mathcal{D}\left(T^{2} x, T^{2} y\right)\right)$ $\geq F_{2}(\mathcal{D}(x, y))+\tau$ or $F_{1}(\mathcal{D}(T x, T y)) \geq F_{1}(\mathcal{D}(x, y))+\tau$.

Definition 3.2 Let $(X, \mathcal{D})$ be a metric space. A mapping $T: X \mapsto X$ is said to be double $F$-expanding of type II, if there exist $\tau>0$ and $F_{1}, F_{2} \in \mathcal{F}$ such that, for all $x, y \in X$,

$$
\begin{aligned}
\mathcal{D}\left(T^{2} x, T^{2} y\right)>0 \quad \text { and } \quad \mathcal{D}(T x, T y)>0 \\
\text { imply } \min \left\{F_{2}\left(\mathcal{D}\left(T^{2} x, T^{2} y\right)\right), F_{1}(\mathcal{D}(T x, T y))\right\} \\
\geq \alpha_{2} F_{2}(\mathcal{D}(x, y))+\alpha_{1} F_{1}(\mathcal{D}(x, y))+\tau,
\end{aligned}
$$

where either $\alpha_{1}=0$ or $\alpha_{2}=0$ and $\alpha_{1}+\alpha_{2}=1$.

Remark 3.2 For all $x, y \in X$, the double $F$-expanding mapping of type II will deal with one of the following two cases (R1) and (R2):

$$
\begin{aligned}
& \min \left\{F_{2}\left(\mathcal{D}\left(T^{2} x, T^{2} y\right)\right), F_{1}(\mathcal{D}(T x, T y))\right\} \geq F_{2}(\mathcal{D}(x, y))+\tau, \\
& \min \left\{F_{2}\left(\mathcal{D}\left(T^{2} x, T^{2} y\right)\right), F_{1}(\mathcal{D}(T x, T y))\right\} \geq F_{1}(\mathcal{D}(x, y))+\tau .
\end{aligned}
$$

For some, $x, y \in X$, (R1) further yields $F_{2}\left(\mathcal{D}\left(T^{2} x, T^{2} y\right)\right) \geq F_{2}(\mathcal{D}(x, y))+\tau$, or, $F_{1}(\mathcal{D}(T x$, $T y)) \geq F_{2}(\mathcal{D}(x, y))+\tau$.

Similarly, for some $x, y \in X$, (R2) yields $F_{2}\left(\mathcal{D}\left(T^{2} x, T^{2} y\right)\right) \geq F_{1}(\mathcal{D}(x, y))+\tau$, or, $F_{1}(\mathcal{D}(T x$, $T y)) \geq F_{1}(\mathcal{D}(x, y))+\tau$.

Next, we introduce triple $F$-expanding mappings.

Definition 3.3 Let $(X, \mathcal{D})$ be a metric space. A mapping $T: X \mapsto X$ is said to be a triple $F$-expanding mapping, if there exist $\tau>0$ and $F, F_{1}, F_{2} \in \mathcal{F}$ such that, for all $x, y \in X$, we have

$$
\begin{aligned}
& \mathcal{D}\left(T^{2} x, T^{2} y\right)>0 \quad \text { and } \quad \mathcal{D}(T x, T y)>0 \\
& \text { imply } \min \left\{F_{2}\left(\mathcal{D}\left(T^{2} x, T^{2} y\right)\right), F_{1}(\mathcal{D}(T x, T y))\right\} \geq F(\mathcal{D}(x, y))+\tau .
\end{aligned}
$$


Example 3.1 Take $F_{1}(\alpha)=\ln \alpha$ and $F_{2}(\alpha)=\ln k \alpha, k>0$. Then $F_{1}$ and $F_{2} \in \mathcal{F}$.

The double $F$-expanding mapping of type I will take the form

$$
\min \left\{\ln k\left(\mathcal{D}\left(T^{2} x, T^{2} y\right)\right), \ln (\mathcal{D}(T x, T y))\right\} \geq \alpha_{2} \ln k(\mathcal{D}(x, y))+\alpha_{1} \ln (\mathcal{D}(x, y))+\tau
$$

Condition (F1) allows us to write

$$
\ln \min \left\{k \mathcal{D}\left(T^{2} x, T^{2} y\right), \mathcal{D}(T x, T y)\right) \geq \ln k^{\alpha_{2}}(\mathcal{D}(x, y))^{\alpha_{1}+\alpha_{2}}+\tau
$$

By the assumption of the definition, we have $\alpha_{1}+\alpha_{2}=1$, so

$$
\min \left\{k \mathcal{D}\left(T^{2} x, T^{2} y\right), \mathcal{D}(T x, T y)\right) \geq e^{\tau} k^{\alpha_{2}} \mathcal{D}(x, y)
$$

Note that if we suppose (as a particular case) that, for all $x, y \in X, \mathcal{D}(T x, T y)<k \mathcal{D}\left(T^{2} x, T^{2} y\right)$, then we have $\mathcal{D}(T x, T y) \geq e^{\tau} \mathcal{D}(x, y)$ with $\alpha_{2}=0$. That is, $T$ is an expanding mapping. Further, if for all $x, y \in X, \mathcal{D}(T x, T y)>k \mathcal{D}\left(T^{2} x, T^{2} y\right)$, then we have $\mathcal{D}\left(T^{2} x, T^{2} y\right) \geq e^{\tau} \mathcal{D}(x, y)$. Hence, $T$ is neither a contraction nor an expanding mapping.

Example 3.2 Take $F_{1}(\alpha)=\ln \alpha$ and $F_{2}(\alpha)=\ln k \alpha, k>1$. Then $F_{1}, F_{2} \in \mathcal{F}$.

Then, by the definition of a double $F$-expanding mapping of type II, for all $x, y \in X$, we have

$$
\min \left\{F_{2}\left(\mathcal{D}\left(T^{2} x, T^{2} y\right)\right), F_{1}(\mathcal{D}(T x, T y))\right\} \geq \alpha_{2} F_{2}(\mathcal{D}(x, y))+\alpha_{1} F_{1}(\mathcal{D}(x, y))+\tau
$$

Conditions (R1) and (R2) allow us to write

$$
\begin{aligned}
& \min \left\{F_{2}\left(\mathcal{D}\left(T^{2} x, T^{2} y\right)\right), F_{1}(\mathcal{D}(T x, T y))\right\} \geq F_{1}(\mathcal{D}(x, y))+\tau \\
& \text { or } \\
& \min \left\{F_{2}\left(\mathcal{D}\left(T^{2} x, T^{2} y\right)\right), F_{1}(\mathcal{D}(T x, T y))\right\} \geq F_{2}(\mathcal{D}(x, y))+\tau
\end{aligned}
$$

Relation (4) yields that

$$
\min \left\{\ln \left(k \mathcal{D}\left(T^{2} x, T^{2} y\right)\right), \ln (\mathcal{D}(T x, T y))\right\} \geq \ln (\mathcal{D}(x, y))+\tau
$$

Condition (F1) allows us to write

$$
\ln \min \left\{k \mathcal{D}\left(T^{2} x, T^{2} y\right), \mathcal{D}(T x, T y)\right\} \geq \ln (\mathcal{D}(x, y))+\tau
$$

so that

$$
\min \left\{k \mathcal{D}\left(T^{2} x, T^{2} y\right), \mathcal{D}(T x, T y)\right\} \geq e^{\tau}(\mathcal{D}(x, y))
$$

Relation (5) implies that

$$
\min \left\{\ln \left(k \mathcal{D}\left(T^{2} x, T^{2} y\right)\right), \ln (\mathcal{D}(T x, T y))\right\} \geq \ln (k \mathcal{D}(x, y))+\tau
$$


With the usage of condition (F1), we can write

$$
\min \left\{k \mathcal{D}\left(T^{2} x, T^{2} y\right), \mathcal{D}(T x, T y)\right\} \geq e^{\tau}(k \mathcal{D}(x, y)) .
$$

One may observe that, for all $x, f(x)=y \in X$, relations (6) and (7) produce the sequences in which the iterates of $T$ may have several combinations of expansions and contractions.

Example 3.3 Given $F_{2}(\alpha)=\ln k_{2} \alpha, F_{1}(\alpha)=\ln k_{1} \alpha$, and $F(\alpha)=\ln k \alpha$, where $k, k_{1}, k_{2}>0$, then $F, F_{1}, F_{2} \in \mathcal{F}$.

Then, by the definition of a triple $F$-expanding mapping, we have for all $x, y \in X$

$$
\begin{aligned}
& \min \left\{F_{2}\left(\mathcal{D}\left(T^{2} x, T^{2} y\right)\right), F_{1}(\mathcal{D}(T x, T y))\right\} \geq F(\mathcal{D}(x, y))+\tau \\
& \text { or } \\
& \min \left\{\ln \left(k_{2} \mathcal{D}\left(T^{2} x, T^{2} y\right)\right), \ln \left(k_{1} \mathcal{D}(T x, T y)\right)\right\} \geq \ln (k \mathcal{D}(x, y))+\tau .
\end{aligned}
$$

Condition (F1) allows us to write

$$
\begin{aligned}
& \ln \min \left\{k_{2} \mathcal{D}\left(T^{2} x, T^{2} y\right), k_{1} \mathcal{D}(T x, T y)\right) \geq \ln k \mathcal{D}(x, y)+\tau \\
& \text { or } \\
& \min \left\{k_{2} \mathcal{D}\left(T^{2} x, T^{2} y\right), k_{1} \mathcal{D}(T x, T y)\right) \geq e^{\tau} k \mathcal{D}(x, y)+\tau .
\end{aligned}
$$

Then either $k_{2}\left(\mathcal{D}\left(T^{2} x, T^{2} y\right)\right) \geq k e^{\tau}(\mathcal{D}(x, y))$ or $k_{1}(\mathcal{D}(T x, T y)) \geq k e^{\tau}(\mathcal{D}(x, y))$.

We can define $k_{2}=2 \alpha_{2}, k_{1}=2 \alpha_{1}, k_{1}+k_{2}=2$, where $\alpha_{1}+\alpha_{2}=1$.

So that we have either

$$
\alpha_{2}\left(\mathcal{D}\left(T^{2} x, T^{2} y\right)\right) \geq \frac{1}{2} k e^{\tau}(\mathcal{D}(x, y))
$$

or

$$
\alpha_{1}(\mathcal{D}(T x, T y)) \geq \frac{1}{2} k e^{\tau}(\mathcal{D}(x, y)) .
$$

Both of the above inequalities can be written as

$$
\alpha_{2}\left(\mathcal{D}\left(T^{2} x, T^{2} y\right)\right)+\alpha_{1}(\mathcal{D}(T x, T y)) \geq k e^{\tau}(\mathcal{D}(x, y)) .
$$

That is a reversal of a mean Lipschitzian mapping, and so the fixed point of (3) will be the fixed point of $T$.

Theorem 3.1 Let $(X, \mathcal{D})$ be a complete metric space. Suppose that a surjective continuous mapping $T: X \mapsto X$ is a double F-expanding mapping of type $I$, and for all $t_{1}, t_{2} \in \mathbb{R}_{+}$, there are $\sigma>0$ and $\tau>\sigma$ such that

$$
F_{2}\left(t_{2}\right)<F_{1}\left(t_{1}\right) \quad \text { implies } F_{1}\left(t_{1}\right) \leq F_{2}\left(t_{2}\right)+\sigma \text {. }
$$

Then $T$ has a unique fixed point in $X$, and for every $x_{0} \in X$, the sequence $\left\{T^{m} x_{0}\right\}_{m=1}^{\infty}$ converges in $X$. 
Proof Consider a sequence $\left\{x_{1}, x_{2}, \ldots\right\}$ such that, for any $x_{0} \in X$, we have $x_{m+1}=T x_{m}=$ $T^{m+1} x_{0}$ for all $m \in \mathbb{N}_{0}$. If, for some $m \in \mathbb{N}, \mathcal{D}\left(x_{m}, T x_{m}\right)=0, T$ admits a fixed point.

Let $\mathcal{D}\left(x_{m}, T x_{m}\right)=\mathcal{D}\left(T x_{m-1}, T x_{m}\right)>0$ for all $m \in \mathbb{N}$.

We will prove that $\lim _{m \rightarrow \infty} \mathcal{D}\left(x_{m}, T x_{m}\right)=0$.

For any $m \in \mathbb{N}$, we can write

$$
\begin{gathered}
\min \left\{F_{2}\left(\mathcal{D}\left(T^{2} x_{m-1}, T^{2} x_{m}\right)\right), F_{1}\left(\mathcal{D}\left(T x_{m-1}, T x_{m}\right)\right)\right\} \\
\geq \alpha_{2} F_{2}\left(\mathcal{D}\left(x_{m-1}, x_{m}\right)\right)+\alpha_{1} F_{1}\left(\mathcal{D}\left(x_{m-1}, x_{m}\right)\right)+\tau .
\end{gathered}
$$

Now, we will discuss the two possible cases $(C)$ and (D):

$$
\begin{aligned}
& F_{1}\left(\mathcal{D}\left(T x_{m-1}, T x_{m}\right)\right)=\min \left\{F_{2}\left(\mathcal{D}\left(T^{2} x_{m-1}, T^{2} x_{m}\right)\right), F_{1}\left(\mathcal{D}\left(T x_{m-1}, T x_{m}\right)\right)\right\} \\
& F_{2}\left(\mathcal{D}\left(T^{2} x_{m-1}, T^{2} x_{m}\right)\right)=\min \left\{F_{2}\left(\mathcal{D}\left(T^{2} x_{m-1}, T^{2} x_{m}\right)\right), F_{1}\left(\mathcal{D}\left(T x_{m-1}, T x_{m}\right)\right)\right\} .
\end{aligned}
$$

If (C) holds, then by the conditions of Definition 3.1, inequality (8) will take the form

$$
\min \left\{F_{2}\left(\mathcal{D}\left(T^{2} x_{m-1}, T^{2} x_{m}\right)\right), F_{1}\left(\mathcal{D}\left(T x_{m-1}, T x_{m}\right)\right)\right\} \geq F_{1}\left(\mathcal{D}\left(x_{m-1}, x_{m}\right)\right)+\tau
$$

Relation (9) further yields

$$
\begin{aligned}
& \min \left\{F_{2}\left(\mathcal{D}\left(T^{2} x_{m-1}, T^{2} x_{m}\right)\right), F_{1}\left(\mathcal{D}\left(T x_{m-1}, T x_{m}\right)\right)\right\} \\
& \quad>\min \left\{F_{2}\left(\mathcal{D}\left(T x_{m-1}, T x_{m}\right)\right), F_{1}\left(\mathcal{D}\left(x_{m-1}, x_{m}\right)\right)\right\}+\tau .
\end{aligned}
$$

Therefore, the possible existence of (C) implies the existence of (10).

Similarly, if (D) holds, inequality (8) will take the form

$$
\min \left\{F_{2}\left(\mathcal{D}\left(T^{2} x_{m-1}, T^{2} x_{m}\right)\right), F_{1}\left(\mathcal{D}\left(T x_{m-1}, T x_{m}\right)\right)\right\} \geq F_{2}\left(\mathcal{D}\left(x_{m-1}, x_{m}\right)\right)+\tau
$$

If $F_{2}\left(\mathcal{D}\left(x_{m-1}, x_{m}\right)\right) \geq F_{1}\left(\mathcal{D}\left(x_{m-1}, x_{m}\right)\right)$, then (11) can be written as

$$
\min \left\{F_{2}\left(\mathcal{D}\left(T^{2} x_{m-1}, T^{2} x_{m}\right)\right), F_{1}\left(\mathcal{D}\left(T x_{m-1}, T x_{m}\right)\right)\right\} \geq F_{1}\left(\mathcal{D}\left(x_{m-1}, x_{m}\right)\right)+\tau
$$

If $F_{2}\left(\mathcal{D}\left(x_{m-1}, x_{m}\right)\right)<F_{1}\left(\mathcal{D}\left(x_{m-1}, x_{m}\right)\right)$, then condition (A) allows us to write

$$
\min \left\{F_{2}\left(\mathcal{D}\left(T^{2} x_{m-1}, T^{2} x_{m}\right)\right), F_{1}\left(\mathcal{D}\left(T x_{m-1}, T x_{m}\right)\right)\right\} \geq F_{1}\left(\mathcal{D}\left(x_{m-1}, x_{m}\right)\right)-\sigma+\tau .
$$

Combining inequalities (12) and (13),

$$
\min \left\{F_{2}\left(\mathcal{D}\left(T^{2} x_{m-1}, T^{2} x_{m}\right)\right), F_{1}\left(\mathcal{D}\left(T x_{m-1}, T x_{m}\right)\right)\right\} \geq F_{1}\left(\mathcal{D}\left(x_{m-1}, x_{m}\right)\right)+\eta_{m} \sigma+\tau,
$$

where

$$
\eta_{m}=\left\{\begin{array}{ll}
-1 & \text { if } F_{2}(t)<F_{1}(t), \\
0 & \text { if } F_{2}(t) \geq F_{1}(t),
\end{array} \quad t \in \mathbb{R}_{+} .\right.
$$


The above inequality can be written as

$$
\begin{aligned}
& \min \left\{F_{2}\left(\mathcal{D}\left(T^{2} x_{m-1}, T^{2} x_{m}\right)\right), F_{1}\left(\mathcal{D}\left(T x_{m-1}, T x_{m}\right)\right)\right\} \\
& \quad \geq \min \left\{F_{2}\left(\mathcal{D}\left(T x_{m-1}, T x_{m}\right)\right), F_{1}\left(\mathcal{D}\left(x_{m-1}, x_{m}\right)\right)\right\}+\eta_{m} \sigma+\tau .
\end{aligned}
$$

Therefore, the existence of (D) implies the existence of inequality (14).

Both cases (C) and (D) yield inequalities (10) and (13) that can be written in the combined form as follows:

$$
\begin{aligned}
& \min \left\{F_{2}\left(\mathcal{D}\left(T^{2} x_{m-1}, T^{2} x_{m}\right)\right), F_{1}\left(\mathcal{D}\left(T x_{m-1}, T x_{m}\right)\right)\right\} \\
& \quad \geq \min \left\{F_{2}\left(\mathcal{D}\left(T x_{m-1}, T x_{m}\right)\right), F_{1}\left(\mathcal{D}\left(x_{m-1}, x_{m}\right)\right)\right\}+\eta_{m} \varsigma_{m} \sigma+\tau,
\end{aligned}
$$

where $\varsigma_{m}$ is either 0 or 1 .

The above inequality can also be written as

$$
\begin{aligned}
& \min \left\{F_{2}\left(\mathcal{D}\left(T^{2} x_{m-1}, T^{2} x_{m}\right)\right), F_{1}\left(\mathcal{D}\left(T x_{m-1}, T x_{m}\right)\right)\right\} \\
& \quad \geq \min \left\{F_{2}\left(\mathcal{D}\left(T^{2} x_{m-2}, T^{2} x_{m-1}\right)\right), F_{1}\left(\mathcal{D}\left(T x_{m-2}, T x_{m-1}\right)\right)\right\}+\eta_{m} \varsigma_{m} \sigma+\tau .
\end{aligned}
$$

Repeating this process, we have

$$
\begin{aligned}
& \min \left\{F_{2}\left(\mathcal{D}\left(T^{2} x_{m-1}, T^{2} x_{m}\right)\right), F_{1}\left(\mathcal{D}\left(T x_{m-1}, T x_{m}\right)\right)\right\} \\
& \quad \geq \min \left\{F_{2}\left(\mathcal{D}\left(T^{2} x_{m-3}, T^{2} x_{m-2}\right)\right), F_{1}\left(\mathcal{D}\left(T x_{m-3}, T x_{m-2}\right)\right)\right\} \\
& \eta_{m-1} \varsigma_{m-1} \sigma+\eta_{m} \varsigma_{m} \sigma-2 \tau \\
& \vdots \\
& \min \left\{F_{2}\left(\mathcal{D}\left(T^{2} x_{m-1}, T^{2} x_{m}\right)\right), F_{1}\left(\mathcal{D}\left(T x_{m-1}, T x_{m}\right)\right)\right\} \\
& \quad \geq \min \left\{F_{2}\left(\mathcal{D}\left(T^{2} x_{1}, T^{2} x_{0}\right)\right), F_{1}\left(\mathcal{D}\left(T x_{1}, T x_{0}\right)\right)\right\}+\sum_{j=1}^{m} \eta_{j} \varsigma_{j} \sigma+m \tau
\end{aligned}
$$

or

$$
\begin{aligned}
& \lim _{m \rightarrow \infty} \min \left\{F_{2}\left(\mathcal{D}\left(T^{2} x_{m-1}, T^{2} x_{m}\right)\right), F_{1}\left(\mathcal{D}\left(T x_{m-1}, T x_{m}\right)\right)\right\}-\sum_{j=1}^{m} \eta_{j} \varsigma_{j} \sigma-m \tau \\
& \quad \geq \min \left\{F_{2}\left(\mathcal{D}\left(T^{2} x_{1}, T^{2} x_{0}\right)\right), F_{1}\left(\mathcal{D}\left(T x_{1}, T x_{0}\right)\right)\right\} .
\end{aligned}
$$

Next, we will show that $T$ is bijective.

Let $T x=T y$, so that $\mathcal{D}(T x, T y)=0$.

If $\mathcal{D}(x, y)>0$, we have

$$
\min \left\{F_{2}\left(\mathcal{D}\left(T^{2} x, T^{2} y\right)\right), F_{1}(\mathcal{D}(T x, T y))\right\} \geq \alpha_{2} F_{2}(\mathcal{D}(x, y))+\alpha_{1} F_{1}(\mathcal{D}(x, y))+t
$$

So,

$$
\min \left\{F_{2}(0), F_{1}(0)\right) \geq \alpha_{2} F_{2}(\mathcal{D}(x, y))+\alpha_{1} F_{1}(\mathcal{D}(x, y))+t,
$$


which implies that

$$
F_{2}(0) \geq F_{2}(\mathcal{D}(x, y))+t, \quad \text { or } \quad F_{1}(0) \geq F_{1}(\mathcal{D}(x, y))+t
$$

Both of the above inequalities are contradictions, and so we have $\mathcal{D}(x, y)=0$ if and only if $x=y$. Therefore, $T$ is bijective.

Consider a mapping $\Omega$ such that $T \Omega=\Omega T=I$, where $I$ is the identity mapping.

For a sequence $\left\{x_{1}, x_{2}, \ldots, x_{m+3}, \ldots\right\}=\left\{x_{1}, T x_{1}, \ldots T^{m+2} x_{1}, \ldots\right\}$, we can choose $x_{m+3}=u_{1}$, so that $\Omega^{m+2} u_{1}=u_{m+3}=x_{1}, \Omega^{m+1} u_{1}=u_{m+2}=x_{2}, \Omega^{m} u_{1}=u_{m+1}=x_{3}, \Omega^{m-1} u_{1}=u_{m}=x_{4}$. Moreover, $x_{m+3}=u_{1}$. It implies that $x_{m+2}=u_{2}, x_{m+1}=u_{3}, x_{m}=u_{4}$. Hence, inequality (20) yields

$$
\begin{aligned}
& \min \left\{F_{2}\left(\mathcal{D}\left(u_{3}, u_{2}\right)\right), F_{1}\left(\mathcal{D}\left(u_{4}, u_{3}\right)\right)\right\}-\lim _{m \rightarrow \infty} \sum_{j=1}^{m} \eta_{j} \varsigma_{j} \sigma-m \tau \\
& \geq \lim _{m \rightarrow \infty} \min \left\{F_{2}\left(\mathcal{D}\left(u_{m+2}, u_{m+1}\right)\right), F_{1}\left(\mathcal{D}\left(u_{m+3}, u_{m+2}\right)\right)\right\}
\end{aligned}
$$

or

$$
\begin{aligned}
& \min \left\{F_{2}\left(\mathcal{D}\left(\Omega^{2} u_{1}, \Omega^{2} u_{0}\right)\right), F_{1}\left(\mathcal{D}\left(\Omega^{3} u_{1}, \Omega^{3} u_{0}\right)\right)\right\}-\lim _{m \rightarrow \infty} \sum_{j=1}^{m} \eta_{j} \varsigma_{j} \sigma-m \tau \\
& \quad \geq \lim _{m \rightarrow \infty} \min \left\{F_{2}\left(\mathcal{D}\left(\Omega^{2} u_{m}, \Omega^{2} u_{m-1}\right)\right), F_{1}\left(\mathcal{D}\left(\Omega^{3} u_{m}, \Omega^{3} u_{m-1}\right)\right)\right\} .
\end{aligned}
$$

Since $\sigma<\tau$, we have

$$
\lim _{m \rightarrow \infty} \min \left\{F_{2}\left(\mathcal{D}\left(\Omega^{2} u_{m}, \Omega^{2} u_{m-1}\right)\right), F_{1}\left(\mathcal{D}\left(\Omega^{3} u_{m}, \Omega^{3} u_{m-1}\right)\right)\right\}=-\infty
$$

Now, equation (16) implies that

$$
\begin{aligned}
& \lim _{m \rightarrow \infty} F_{2}\left(\mathcal{D}\left(\Omega^{2} u_{m-1}, \Omega^{2} u_{m}\right)\right)=-\infty \\
& \text { or } \\
& \lim _{m \rightarrow \infty} F_{1}\left(\mathcal{D}\left(\Omega^{3} u_{m-1}, \Omega^{3} u_{m}\right)\right)=-\infty .
\end{aligned}
$$

Condition (F2) among (E) yields that

$$
\lim _{m \rightarrow \infty} \mathcal{D}\left(\Omega^{2} u_{m-1}, \Omega^{2} u_{m}\right)=0
$$

or equivalently,

$$
\lim _{m \rightarrow \infty} \mathcal{D}\left(\Omega^{2} u_{m-1}, \Omega^{2} u_{m}\right)=\lim _{m \rightarrow \infty} \mathcal{D}\left(u_{m+1}, \Omega u_{m+1}\right)=\lim _{m \rightarrow \infty} \mathcal{D}\left(u_{m}, \Omega u_{m}\right)=0
$$

Condition (F2) yields

$$
\lim _{m \rightarrow \infty} \mathcal{D}\left(\Omega^{3} u_{m-1}, \Omega^{3} u_{m}\right)=\lim _{m \rightarrow \infty} \mathcal{D}\left(u_{m+2}, \Omega u_{m+2}\right)=\lim _{m \rightarrow \infty} \mathcal{D}\left(u_{m}, \Omega u_{m}\right)=0
$$


Therefore, from (21) we get

$$
\lim _{m \rightarrow \infty} \mathcal{D}\left(u_{m}, \Omega u_{m}\right)=0
$$

Now, we will prove that the sequence $\left\{u_{m}\right\}_{m=1}^{\infty}$ is a Cauchy sequence. On the contrary, suppose that there exist $\varepsilon>0$ and sequences $\{\mathfrak{g}(m)\}_{n=1}^{\infty}$ and $\{\mathfrak{h}(m)\}_{m=1}^{\infty}$ of natural numbers such that

$$
\begin{array}{ll}
\mathfrak{g}(m)>\mathfrak{h}(m)>m, \quad & \mathcal{D}\left(u_{\mathfrak{g}(m)}, u_{\mathfrak{h}(m)}\right) \geq \varepsilon, \\
\mathcal{D}\left(u_{\mathfrak{g}(m)-1}, u_{\mathfrak{h}(m)}\right)<\varepsilon & \text { for all } m \in \mathbb{N} .
\end{array}
$$

We further suppose that $\mathfrak{h}(m)$ is greater than $\mathfrak{g}(m)$ by $\mathfrak{l}(m)$.

Now, we can write

$$
\begin{aligned}
\varepsilon & \leq \mathcal{D}\left(u_{\mathfrak{g}(m)}, u_{\mathfrak{h}(m)}\right) \\
& \leq \mathcal{D}\left(u_{\mathfrak{g}(m)}, u_{\mathfrak{g}(m)-1}\right)+\mathcal{D}\left(u_{\mathfrak{g}(m)-1}, u_{\mathfrak{h}(m)}\right) \\
& <\mathcal{D}\left(u_{\mathfrak{g}(m)}, u_{\mathfrak{g}(m)-1}\right)+\varepsilon \\
& =\mathcal{D}\left(u_{\mathfrak{g}(m)-1}, T u_{\mathfrak{g}(m)-1}\right)+\varepsilon .
\end{aligned}
$$

That is,

$$
\varepsilon \leq \mathcal{D}\left(u_{\mathfrak{g}(m)}, u_{\mathfrak{h}(m)}\right)<\mathcal{D}\left(u_{\mathfrak{g}(m)-1}, T u_{\mathfrak{g}(m)-1}\right)+\varepsilon .
$$

The above inequality along with (19) yields

$$
\lim _{m \rightarrow \infty} \mathcal{D}\left(u_{\mathfrak{g}(m)}, u_{\mathfrak{h}(m)}\right)=\varepsilon
$$

Further, from (17) there exists $N \in \mathbb{N}$ such that

$$
\mathcal{D}\left(u_{\mathfrak{g}(m)}, T u_{\mathfrak{g}(m)}\right)<\frac{\varepsilon}{4} \quad \text { and } \quad \mathcal{D}\left(u_{\mathfrak{h}(m)}, T u_{\mathfrak{h}(m)}\right)<\frac{\varepsilon}{4} \quad \text { for all } m \geq N
$$

Next, we claim that

$$
\mathcal{D}\left(u_{\mathfrak{g}(m)}, u_{\mathfrak{h}(m)}\right)=\mathcal{D}\left(u_{\mathfrak{g}(m)+1}, u_{\mathfrak{h}(m)+1}\right)>0 \quad \text { for all } m \geq N .
$$

On the contrary, suppose that there exists $r \geq N$ such that

$$
\mathcal{D}\left(u_{\mathfrak{g}(r)+1}, u_{\mathfrak{h}(r)+1}\right)=0 .
$$

It follows from (18), (20), and (22) that

$$
\begin{aligned}
\varepsilon & \leq \mathcal{D}\left(u_{\mathfrak{g}(r)}, u_{\mathfrak{h}(r)}\right) \leq \mathcal{D}\left(u_{\mathfrak{g}(r)}, u_{\mathfrak{g}(r)+1}\right)+\mathcal{D}\left(u_{\mathfrak{g}(r)+1}, u_{\mathfrak{h}(r)}\right) \\
& \leq \mathcal{D}\left(u_{\mathfrak{g}(r)}, u_{\mathfrak{g}(r)+1}\right)+\mathcal{D}\left(u_{\mathfrak{g}(r)+1}, u_{\mathfrak{h}(r)+1}\right)+\mathcal{D}\left(u_{\mathfrak{h}(r)+1}, u_{\mathfrak{h}(r)}\right) \\
& =\mathcal{D}\left(u_{\mathfrak{g}(r)}, T u_{\mathfrak{g}(r)}\right)+\mathcal{D}\left(u_{\mathfrak{g}(r)+1}, u_{\mathfrak{h}(r)+1}\right)+\mathcal{D}\left(u_{\mathfrak{h}(r)}, T u_{\mathfrak{h}(r)}\right)
\end{aligned}
$$




$$
<\frac{\varepsilon}{4}+0+\frac{\varepsilon}{4}=\frac{\varepsilon}{2}
$$

That is a contradiction.

Next, we suppose that, for some $x_{\mathfrak{b}(m)}, x_{\mathfrak{r}(m)} \in X$, we have $u_{\mathfrak{g}(m)}=x_{\mathfrak{b}(m)}$ and $u_{\mathfrak{h}(m)}=x_{\mathfrak{r}(m)}$ such that $u_{\mathfrak{g}(m)-1}=T x_{\mathfrak{b}(m)}, u_{\mathfrak{h}(m)-1}=T x_{\mathfrak{r}(m)}, u_{\mathfrak{g}(m)-2}=T^{2} x_{\mathfrak{b}(m)}, u_{\mathfrak{h}(m)-2}=T^{2} x_{\mathfrak{r}(m)}$.

Therefore, relation (21) with the assumption of the theorem gives

$$
\begin{gathered}
\mathcal{D}\left(x_{\mathfrak{b}(m)}, x_{\mathfrak{r}(m)}\right)=\mathcal{D}\left(x_{\mathfrak{b}(m)+1}, x_{\mathfrak{r}(m)+1}\right)>0 \text { implies } \\
\min \left\{F_{2}\left(\mathcal{D}\left(T^{2} x_{\mathfrak{b}(m)}, T^{2} x_{\mathfrak{r}(m)}\right)\right), F_{1}\left(\mathcal{D}\left(T x_{\mathfrak{b}(m)}, T x_{\mathfrak{r}(m)}\right)\right)\right\} \\
\geq \alpha_{2} F_{2}\left(x_{\mathfrak{b}(m)}, x_{\mathfrak{r}(m)}\right)+\alpha_{1} F_{1}\left(\mathcal{D}\left(x_{\mathfrak{b}(m)}, x_{\mathfrak{r}(m)}\right)\right)+\tau .
\end{gathered}
$$

Now, we will deal with two possible cases of (23):

$$
F_{2}\left(\mathcal{D}\left(T^{2} x_{\mathfrak{b}(m)}, T^{2} x_{\mathfrak{r}(m)}\right)\right) \geq F_{2}\left(x_{\mathfrak{b}(m)}, x_{\mathfrak{r}(m)}\right)+\tau
$$

or

$$
F_{1}\left(\mathcal{D}\left(T x_{\mathfrak{b}(m)}, T x_{\mathfrak{r}(m)}\right)\right) \geq F_{1}\left(x_{\mathfrak{b}(m)}, x_{\mathfrak{r}(m)}\right)+\tau .
$$

Both of the above inequalities will take the form

$$
\begin{aligned}
& F_{2}\left(\mathcal{D}\left(\Omega^{2} u_{\mathfrak{g}(m-4)}, \Omega^{2} x_{\mathfrak{h}(m-4)}\right)\right) \geq F_{2}\left(\Omega^{4} u_{\mathfrak{g}(m-4)}, \Omega^{4} u_{\mathfrak{h}(m-4)}\right)+\tau \\
& \text { or } \\
& F_{1}\left(\mathcal{D}\left(\Omega^{3} q_{\mathfrak{g}(m-4)}, \Omega^{3} h_{\mathfrak{r}(m-4)}\right)\right) \geq F_{1}\left(\Omega^{4} u_{\mathfrak{g}(m)}, \Omega^{4} u_{\mathfrak{h}(m)}\right)+\tau .
\end{aligned}
$$

So that we have the following contradictions: $F_{2}(\varepsilon) \geq F_{2}(\varepsilon)+\tau$ or $F_{1}(\varepsilon) \geq F_{1}(\varepsilon)+\tau$.

Therefore, $\left\{u_{m}\right\}_{n=1}^{\infty}$ is a Cauchy sequence. The completeness of $(X, \mathcal{D})$ proves that $\left\{u_{m}\right\}_{n=1}^{\infty}$ converges to some point $u^{*}$ in $X$. Now, the continuity of $\Omega$ implies that

$$
\mathcal{D}(\Omega u, u)=\lim _{m \rightarrow \infty} \mathcal{D}\left(\Omega u_{m}, u_{m}\right)=\lim _{m \rightarrow \infty} \mathcal{D}\left(u_{m+1}, u_{m}\right)=\mathcal{D}\left(u^{*}, u^{*}\right)=0
$$

Therefore, $\Omega$ has a fixed point $u^{*}$ in $X$ and $\Omega u^{*}=u^{*}$ so that $u^{*}=T u^{*}$. Now, for the uniqueness, let us suppose that $T$ has more than one fixed point. That is, there exist two distinct $u, v \in X$ such that $T u=u \neq v=T v$.

Therefore, $\mathcal{D}(u, v)=\mathcal{D}(T u, T v)=\mathcal{D}\left(T^{2} u, T^{2} v\right)>0$ with relation (1) implies that either

$$
F_{1}(\mathcal{D}(T u, T v)) \geq F_{1}(\mathcal{D}(u, v))+\tau>F_{1}(\mathcal{D}(u, v))=F_{1}(\mathcal{D}(T u, T v))
$$

or

$$
F_{2}\left(\mathcal{D}\left(T^{2} u, T^{2} v\right)\right) \geq F_{2}(\mathcal{D}(u, v))+\tau>F_{2}(\mathcal{D}(u, v))=F_{2}\left(\mathcal{D}\left(T^{2} u, T^{2} v\right)\right) .
$$

Both relations (24) and (25) are the contradictions, and so we have a unique fixed point.

Theorem 3.2 Let $(X, \mathcal{D})$ be a complete metric space. Suppose that a surjective continuous mapping $T: X \mapsto X$ is a double F-expanding mapping of type $I I$, and for all $t, t_{1}, t_{2} \in \mathbb{R}_{+}$, 
there is $\sigma>0$ such that $\sigma<\tau$ and

$$
\begin{aligned}
& F_{2}(t)<F_{1}(t) \quad \text { implies } F_{1}(t) \leq F_{2}(t)+\sigma, \\
& F_{1}(t)<F_{2}(t) \quad \text { implies } F_{2}(t) \leq F_{1}(t)+\sigma .
\end{aligned}
$$

Then $T$ has a unique fixed point in $X$, and for every $x_{0} \in X$, the sequence $\left\{T^{m} x_{0}\right\}_{m=1}^{\infty}$ converges to a definite number.

Proof Consider a sequence $\left\{x_{1}, x_{2}, \ldots\right\}$ such that $x_{m+1}=T x_{m}=T^{m+1} x_{0}$ for any $x_{0} \in X$, for all $m \in \mathbb{N}_{0}$. If, for some $m \in \mathbb{N}, \mathcal{D}\left(x_{m}, T x_{m}\right)=0$, $T$ will admit a fixed point. Let $\mathcal{D}\left(x_{m}, T x_{m}\right)=\mathcal{D}\left(T x_{m-1}, T x_{m}\right)>0$ for all $m \in \mathbb{N}$.

If, for all $x, y \in X$, relation (R2) holds, then the analysis of the previous theorem yields

$$
\begin{aligned}
& \min \left\{F_{2}\left(\mathcal{D}\left(T^{2} x_{m-1}, T^{2} x_{m}\right)\right), F_{1}\left(\mathcal{D}\left(T x_{m-1}, T x_{m}\right)\right)\right\} \\
& \quad \geq \min \left\{F_{2}\left(\mathcal{D}\left(T x_{m-1}, T x_{m}\right)\right), F_{1}\left(\mathcal{D}\left(x_{m-1}, x_{m}\right)\right)\right\}+\tau .
\end{aligned}
$$

The above inequality can be written as follows:

$$
\begin{aligned}
& \min \left\{F_{2}\left(\mathcal{D}\left(T^{2} x_{m-1}, T^{2} x_{m}\right)\right), F_{1}\left(\mathcal{D}\left(T x_{m-1}, T x_{m}\right)\right)\right\} \\
& \quad \geq \min \left\{F_{2}\left(\mathcal{D}\left(T^{2} x_{m-2}, T^{2} x_{m-1}\right)\right), F_{1}\left(\mathcal{D}\left(T x_{m-2}, T x_{m-1}\right)\right)\right\}+\tau .
\end{aligned}
$$

Repeating this process, we have

$$
\begin{aligned}
& \min \left\{F_{2}\left(\mathcal{D}\left(T^{2} x_{m-1}, T^{2} x_{m}\right)\right), F_{1}\left(\mathcal{D}\left(T x_{m-1}, T x_{m}\right)\right)\right\} \\
& \quad \geq \min \left\{F_{2}\left(\mathcal{D}\left(T^{2} x_{m-3}, T^{2} x_{m-2}\right)\right), F_{1}\left(\mathcal{D}\left(T x_{m-3}, T x_{m-2}\right)\right)\right\}+2 \tau, \\
& \quad \min \left\{F_{2}\left(\mathcal{D}\left(T^{2} x_{m-1}, T^{2} x_{m}\right)\right), F_{1}\left(\mathcal{D}\left(T x_{m-1}, T x_{m}\right)\right)\right\} \\
& \quad \geq \min \left\{F_{2}\left(\mathcal{D}\left(T^{2} x_{1}, T^{2} x_{0}\right)\right), F_{1}\left(\mathcal{D}\left(T x_{1}, T x_{0}\right)\right)\right\}+m \tau .
\end{aligned}
$$

Now, the analysis similar to the previous theorem yields

$$
\begin{aligned}
& \min \left\{F_{2}\left(\mathcal{D}\left(u_{3}, u_{2}\right)\right), F_{1}\left(\mathcal{D}\left(u_{4}, u_{3}\right)\right)\right\}-m \tau \\
& \quad \geq \lim _{m \rightarrow \infty} \min \left\{F_{2}\left(\mathcal{D}\left(u_{m+2}, u_{m+1}\right)\right), F_{1}\left(\mathcal{D}\left(u_{m+3}, u_{m+2}\right)\right)\right\}
\end{aligned}
$$

or

$$
\begin{aligned}
& \min \left\{F_{2}\left(\mathcal{D}\left(\Omega^{2} u_{1}, \Omega^{2} u_{0}\right)\right), F_{1}\left(\mathcal{D}\left(\Omega^{3} u_{1}, \Omega^{3} u_{0}\right)\right)\right\}-m \tau \\
& \quad \geq \lim _{m \rightarrow \infty} \min \left\{F_{2}\left(\mathcal{D}\left(\Omega^{2} u_{m}, \Omega^{2} u_{m-1}\right)\right), F_{1}\left(\mathcal{D}\left(\Omega^{3} u_{m}, \Omega^{3} u_{m-1}\right)\right)\right\}, \\
& \lim _{m \rightarrow \infty} \min \left\{F_{2}\left(\mathcal{D}\left(\Omega^{2} u_{m}, \Omega^{2} u_{m-1}\right)\right), F_{1}\left(\mathcal{D}\left(\Omega^{3} u_{m}, \Omega^{3} u_{m-1}\right)\right)\right\}=-\infty .
\end{aligned}
$$

If, for all $x, y \in X$, relation (R1) holds, then we can write

$$
\min \left\{F_{2}\left(\mathcal{D}\left(T^{2} x_{m-1}, T^{2} x_{m}\right)\right), F_{1}\left(\mathcal{D}\left(T x_{m-1}, T x_{m}\right)\right)\right\} \geq F_{1}\left(\mathcal{D}\left(x_{m-1}, x_{m}\right)\right)+\eta_{m} \sigma+\tau,
$$


where

$$
\eta_{m}=\left\{\begin{array}{ll}
-1 & \text { if } F_{2}(t)<F_{1}(t), \\
0 & \text { if } F_{2}(t) \geq F_{1}(t),
\end{array} \quad t \in \mathbb{R}_{+} .\right.
$$

The above inequality will take the form

$$
\begin{aligned}
& \min \left\{F_{2}\left(\mathcal{D}\left(T^{2} x_{m-1}, T^{2} x_{m}\right)\right), F_{1}\left(\mathcal{D}\left(T x_{m-1}, T x_{m}\right)\right)\right\} \\
& \quad \geq \min \left\{F_{2}\left(\mathcal{D}\left(T x_{m-1}, T x_{m}\right)\right), F_{1}\left(\mathcal{D}\left(x_{m-1}, x_{m}\right)\right)\right\}+\eta_{m} \sigma+\tau .
\end{aligned}
$$

That can be written as

$$
\begin{aligned}
& \min \left\{F_{2}\left(\mathcal{D}\left(T^{2} x_{m-1}, T^{2} x_{m}\right)\right), F_{1}\left(\mathcal{D}\left(T x_{m-1}, T x_{m}\right)\right)\right\} \\
& \quad \geq \min \left\{F_{2}\left(\mathcal{D}\left(T^{2} x_{m-2}, T^{2} x_{m-1}\right)\right), F_{1}\left(\mathcal{D}\left(T x_{m-2}, T x_{m-1}\right)\right)\right\}+\eta_{m} \sigma+\tau .
\end{aligned}
$$

Repeating this process, we have

$$
\begin{aligned}
\min & \left\{F_{2}\left(\mathcal{D}\left(T^{2} x_{m-1}, T^{2} x_{m}\right)\right), F_{1}\left(\mathcal{D}\left(T x_{m-1}, T x_{m}\right)\right)\right\} \\
\geq & \min \left\{F_{2}\left(\mathcal{D}\left(T^{2} x_{m-3}, T^{2} x_{m-2}\right)\right), F_{1}\left(\mathcal{D}\left(T x_{m-3}, T x_{m-2}\right)\right)\right\} \\
& +\eta_{m-1} \sigma+\eta_{m} \sigma+2 \tau \\
\vdots & \min \left\{F_{2}\left(\mathcal{D}\left(T^{2} x_{m-1}, T^{2} x_{m}\right)\right), F_{1}\left(\mathcal{D}\left(T x_{m-1}, T x_{m}\right)\right)\right\} \\
\geq & \min \left\{F_{2}\left(\mathcal{D}\left(T^{2} x_{1}, T^{2} x_{0}\right)\right), F_{1}\left(\mathcal{D}\left(T x_{1}, T x_{0}\right)\right)\right\}+\sum_{j=1}^{m} \eta_{j} \sigma+m \tau .
\end{aligned}
$$

So that

$$
\begin{aligned}
& \min \left\{F_{2}\left(\mathcal{D}\left(\Omega^{2} u_{1}, \Omega^{2} u_{0}\right)\right), F_{1}\left(\mathcal{D}\left(\Omega^{3} u_{1}, \Omega^{3} u_{0}\right)\right)\right\}-\lim _{m \rightarrow \infty} \sum_{j=1}^{m} \eta_{j} \sigma-m \tau \\
& \quad \geq \lim _{m \rightarrow \infty} \min \left\{F_{2}\left(\mathcal{D}\left(\Omega^{2} u_{m}, \Omega^{2} u_{m-1}\right)\right), F_{1}\left(\mathcal{D}\left(\Omega^{3} u_{m}, \Omega^{3} u_{m-1}\right)\right)\right\} .
\end{aligned}
$$

Since $\sigma<\tau$, we have

$$
\lim _{m \rightarrow \infty} \min \left\{F_{2}\left(\mathcal{D}\left(\Omega^{2} u_{m}, \Omega^{2} u_{m-1}\right)\right), F_{1}\left(\mathcal{D}\left(\Omega^{3} u_{m}, \Omega^{3} u_{m-1}\right)\right)\right\}=-\infty
$$

Therefore, relations (26) and (27) imply that

$$
\lim _{m \rightarrow \infty} F_{2}\left(\mathcal{D}\left(\Omega^{2} u_{m-1}, \Omega^{2} u_{m}\right)\right)=-\infty .
$$

or,

$$
\lim _{m \rightarrow \infty} F_{1}\left(\mathcal{D}\left(\Omega^{3} u_{m-1}, \Omega^{3} u_{m}\right)\right)=-\infty .
$$


Now, using the analysis of the previous theorem, relations (R1) and (R2) yield that

$$
\begin{aligned}
& F_{2}(\varepsilon) \geq F_{2}(\varepsilon)+\tau, \\
& F_{1}(\varepsilon) \geq F_{1}(\varepsilon)+\tau, \\
& F_{2}(\varepsilon) \geq F_{1}(\varepsilon)+\tau, \\
& F_{1}(\varepsilon) \geq F_{2}(\varepsilon)+\tau .
\end{aligned}
$$

As $\tau>0$, relations (28) and (29) are contradictions.

Now, we consider relation (30)

$$
F_{2}(\varepsilon) \geq F_{1}(\varepsilon)+\tau
$$

Using condition (B'), we have

$$
F_{1}(\varepsilon)+\sigma \geq F_{1}(\varepsilon)+\tau \text {. }
$$

It is a contradiction, as $\tau>\sigma$.

Similarly, $F_{1}(\varepsilon) \geq F_{2}(\varepsilon)+\tau$, which is a contradiction: $\sigma \leq \tau$.

The contradictions of relations (28)-(31) prove that $\left\{u_{m}\right\}_{m=1}^{\infty}$ is a Cauchy sequence. The completeness of $(X, \mathcal{D})$ proves that $\left\{u_{m}\right\}_{m=1}^{\infty}$ converges to some point $u^{*}$ in $X$.

Now, the continuity of $\Omega$ implies

$$
\mathcal{D}(\Omega u, u)=\lim _{m \rightarrow \infty} \mathcal{D}\left(\Omega u_{m}, u_{m}\right)=\lim _{m \rightarrow \infty} \mathcal{D}\left(u_{m+1}, u_{m}\right)=\mathcal{D}\left(u^{*}, u^{*}\right)=0
$$

Therefore, $\Omega$ has a fixed point $u^{*}$ in $X$ and $\Omega u^{*}=u^{*}$. So that $u^{*}=T u^{*}$. Now, for uniqueness, let us suppose that $T$ has more than one fixed point. That is, there exist two distinct $u, v \in X$ such that $T u=u \neq v=T v$. Therefore, $\mathcal{D}(u, v)=\mathcal{D}(T u, T v)=\mathcal{D}\left(T^{2} u, T^{2} v\right)>0$, and the assumption of the theorem leads to the following four possibilities:

$$
\begin{gathered}
F_{1}(\mathcal{D}(u, v))=F_{1}(\mathcal{D}(T u, T v)) \geq F_{1}(\mathcal{D}(u, v))+\tau, \\
F_{2}(\mathcal{D}(u, v))=F_{2}\left(\mathcal{D}\left(T^{2} u, T^{2} v\right)\right) \geq F_{2}(\mathcal{D}(u, v))+\tau, \\
F_{2}(\mathcal{D}(u, v))=F_{2}\left(\mathcal{D}\left(T^{2} u, T^{2} v\right)\right) \geq F_{1}(\mathcal{D}(u, v))+\tau \\
\quad \text { implies } \sigma+F_{1}(\mathcal{D}(u, v)) \geq F_{1}(\mathcal{D}(u, v))+\tau, \\
F_{1}(\mathcal{D}(u, v))=F_{1}(\mathcal{D}(T u, T v)) \geq F_{2}(\mathcal{D}(u, v))+\tau \\
\quad \text { implies } \sigma+F_{1}(\mathcal{D}(u, v)) \geq F_{1}(\mathcal{D}(u, v))+\tau .
\end{gathered}
$$

All the four relations (32)-(35) are contradictions, and so we have a unique fixed point.

Theorem 3.3 Let $(X, \mathcal{D})$ be a complete metric space. Suppose that a continuous mapping $T: X \mapsto X$ is a triple F-contraction, and for all $t, t_{1}, t_{2} \in \mathbb{R}_{+}$, there exist $\sigma>0$ and $\tau>\sigma$ such that $F\left(t_{1}\right)<F_{i}\left(t_{2}\right)$ implies $F_{i}\left(t_{2}\right) \leq F\left(t_{1}\right)+\sigma, i=1,2$.

Then $T$ has a unique fixed point in $X$, and for every $x_{0} \in X$, the sequence $\left\{T^{m} x_{0}\right\}_{m=1}^{\infty}$ converges to a definite number. 
Proof Consider a sequence $\left\{x_{1}, x_{2}, \ldots\right\}$ such that $x_{m+1}=T x_{m}=T^{m+1} x_{0}$ for some $x_{0} \in X$ and for all $m \in \mathbb{W}$. If, for some $m \in \mathbb{N}, \mathcal{D}\left(x_{m}, T x_{m}\right)=0, T$ has a fixed point.

Let $\mathcal{D}\left(x_{m}, T x_{m}\right)=\mathcal{D}\left(T x_{m-1}, T x_{m}\right)>0$ for all $m \in \mathbb{N}$.

We will prove that $\lim _{m \rightarrow \infty} \mathcal{D}\left(x_{m}, T x_{m}\right)=0$.

For any $m \in \mathbb{N}$, we have

$$
\min \left\{F_{2}\left(\mathcal{D}\left(T^{2} x_{m-1}, T^{2} x_{m}\right)\right), F_{1}\left(\mathcal{D}\left(T x_{m-1}, T x_{m}\right)\right)\right\} \geq F\left(\mathcal{D}\left(x_{m-1}, x_{m}\right)\right)+\tau
$$

If $F\left(\mathcal{D}\left(x_{m-1}, x_{m}\right)\right) \geq F_{1}\left(\mathcal{D}\left(x_{m-1}, x_{m}\right)\right)$, inequality (36) can be written as follows:

$$
\min \left\{F_{2}\left(\mathcal{D}\left(T^{2} x_{m-1}, T^{2} x_{m}\right)\right), F_{1}\left(\mathcal{D}\left(T x_{m-1}, T x_{m}\right)\right)\right\} \geq F_{1}\left(\mathcal{D}\left(x_{m-1}, x_{m}\right)\right)+\tau
$$

If $F\left(\mathcal{D}\left(x_{m-1}, x_{m}\right)\right)<F_{1}\left(\mathcal{D}\left(x_{m-1}, x_{m}\right)\right)$, condition (A) allows us to write

$$
F_{1}\left(\mathcal{D}\left(x_{m-1}, x_{m}\right)\right) \leq F\left(\mathcal{D}\left(x_{m-1}, x_{m}\right)\right)+\sigma .
$$

Then inequality (36) can be written as

$$
\min \left\{F_{2}\left(\mathcal{D}\left(T^{2} x_{m-1}, T^{2} x_{m}\right)\right), F_{1}\left(\mathcal{D}\left(T x_{m-1}, T x_{m}\right)\right)\right\} \geq F_{1}\left(\mathcal{D}\left(x_{m-1}, x_{m}\right)\right)-\sigma+\tau .
$$

Combining inequalities (37) and (38), we have

$$
\min \left\{F_{2}\left(\mathcal{D}\left(T^{2} x_{m-1}, T^{2} x_{m}\right)\right), F_{1}\left(\mathcal{D}\left(T x_{m-1}, T x_{m}\right)\right)\right\} \geq F_{1}\left(\mathcal{D}\left(x_{m-1}, x_{m}\right)\right)+\varsigma_{i} \sigma+\tau,
$$

where $\varsigma_{i}$ is either 0 or -1 .

Next, we will consider the following two possible cases:

$$
F_{1}\left(\mathcal{D}\left(x_{m-1}, x_{m}\right)\right)=\min \left\{F_{2}\left(\mathcal{D}\left(T x_{m-1}, T x_{m}\right)\right), F_{1}\left(\mathcal{D}\left(x_{m-1}, x_{m}\right)\right)\right\}
$$

or

$$
F_{2}\left(\mathcal{D}\left(T x_{m-1}, T x_{m}\right)\right)=\min \left\{F_{2}\left(\mathcal{D}\left(T x_{m-1}, T x_{m}\right)\right), F_{1}\left(\mathcal{D}\left(x_{m-1}, x_{m}\right)\right)\right\} .
$$

If (40) is true, inequality (39) will take the form

$$
\begin{aligned}
& \min \left\{F_{2}\left(\mathcal{D}\left(T^{2} x_{m-1}, T^{2} x_{m}\right)\right), F_{1}\left(\mathcal{D}\left(T x_{m-1}, T x_{m}\right)\right)\right\} \\
& \quad \geq \min \left\{F_{2}\left(\mathcal{D}\left(T x_{m-1}, T x_{m}\right)\right), F_{1}\left(\mathcal{D}\left(x_{m-1}, x_{m}\right)\right)\right\}+\varsigma_{i} \sigma+\tau .
\end{aligned}
$$

If (41) is true, we have $F_{2}\left(\mathcal{D}\left(T x_{m-1}, T x_{m}\right)\right)<F_{1}\left(\mathcal{D}\left(x_{m-1}, x_{m}\right)\right)$.

So that relation (41) can be written as

$$
\min \left\{F_{2}\left(\mathcal{D}\left(T^{2} x_{m-1}, T^{2} x_{m}\right)\right), F_{1}\left(\mathcal{D}\left(T x_{m-1}, T x_{m}\right)\right)\right\}>F_{2}\left(\mathcal{D}\left(T x_{m-1}, T x_{m}\right)\right)+\varsigma_{i} \sigma+\tau .
$$

Moreover, condition (41) will change the above inequality in the following form:

$$
\min \left\{F_{2}\left(\mathcal{D}\left(T^{2} x_{m-1}, T^{2} x_{m}\right)\right), F_{1}\left(\mathcal{D}\left(T x_{m-1}, T x_{m}\right)\right)\right\}
$$




$$
>\min \left\{F_{2}\left(\mathcal{D}\left(T x_{m-1}, T x_{m}\right)\right), F_{1}\left(\mathcal{D}\left(x_{m-1}, x_{m}\right)\right)\right\}+\varsigma_{i} \sigma+\tau
$$

Combining (42) and (43), we have

$$
\begin{aligned}
& \min \left\{F_{2}\left(\mathcal{D}\left(T^{2} x_{m-1}, T^{2} x_{m}\right)\right), F_{1}\left(\mathcal{D}\left(T x_{m-1}, T x_{m}\right)\right)\right\} \\
& \quad \geq \min \left\{F_{2}\left(\mathcal{D}\left(T x_{m-1}, T x_{m}\right)\right), F_{1}\left(\mathcal{D}\left(x_{m-1}, x_{m}\right)\right)\right\}+\varsigma_{i} \sigma+\tau .
\end{aligned}
$$

That is equivalent to

$$
\begin{aligned}
& \min \left\{F_{2}\left(\mathcal{D}\left(T^{2} x_{m-1}, T^{2} x_{m}\right)\right), F_{1}\left(\mathcal{D}\left(T x_{m-1}, T x_{m}\right)\right)\right\} \\
& \quad \geq \min \left\{F_{2}\left(\mathcal{D}\left(T^{2} x_{m-2}, T^{2} x_{m-1}\right)\right), F_{1}\left(\mathcal{D}\left(T x_{m-2}, T x_{m-1}\right)\right)\right\}+\varsigma_{i} \sigma+\tau .
\end{aligned}
$$

Repeating this process, we have

$$
\begin{aligned}
& \min \left\{F_{2}\left(\mathcal{D}\left(T^{2} x_{m-1}, T^{2} x_{m}\right)\right), F_{1}\left(\mathcal{D}\left(T x_{m-1}, T x_{m}\right)\right)\right\} \\
& \quad \geq \min \left\{F_{2}\left(\mathcal{D}\left(T^{2} x_{m-3}, T^{2} x_{m-2}\right)\right), F_{1}\left(\mathcal{D}\left(T x_{m-3}, T x_{m-2}\right)\right)\right\}+\varsigma_{i-1} \sigma+\varsigma_{i} \sigma+2 \tau \\
& \vdots \\
& \min \left\{F_{2}\left(\mathcal{D}\left(T^{2} x_{m-1}, T^{2} x_{m}\right)\right), F_{1}\left(\mathcal{D}\left(T x_{m-1}, T x_{m}\right)\right)\right\} \\
& \quad \geq \min \left\{F_{2}\left(\mathcal{D}\left(T^{2} x_{1}, T^{2} x_{0}\right)\right), F_{1}\left(\mathcal{D}\left(T x_{1}, T x_{0}\right)\right)\right\}+\sigma \sum_{j=1}^{m} \varsigma_{j}+m \tau .
\end{aligned}
$$

Now, the analysis similar to the previous theorem yields that

$$
\begin{aligned}
& \min \left\{F_{2}\left(\mathcal{D}\left(u_{3}, u_{2}\right)\right), F_{1}\left(\mathcal{D}\left(u_{4}, u_{3}\right)\right)\right\}-\lim _{m \rightarrow \infty} \sum_{j=1}^{m} \varsigma_{j} \sigma-m \tau \\
& \quad \geq \lim _{m \rightarrow \infty} \min \left\{F_{2}\left(\mathcal{D}\left(u_{m+2}, u_{m+1}\right)\right), F_{1}\left(\mathcal{D}\left(u_{m+3}, u_{m+2}\right)\right)\right\}
\end{aligned}
$$

or

$$
\begin{aligned}
& \min \left\{F_{2}\left(\mathcal{D}\left(\Omega^{2} u_{1}, \Omega^{2} u_{0}\right)\right), F_{1}\left(\mathcal{D}\left(\Omega^{3} u_{1}, \Omega^{3} u_{0}\right)\right)\right\}-\lim _{m \rightarrow \infty} \sum_{j=1}^{m} \delta_{j} \sigma-m \tau \\
& \quad \geq \lim _{m \rightarrow \infty} \min \left\{F_{2}\left(\mathcal{D}\left(\Omega^{2} u_{m}, \Omega^{2} u_{m-1}\right)\right), F_{1}\left(\mathcal{D}\left(\Omega^{3} u_{m}, \Omega^{3} u_{m-1}\right)\right)\right\}
\end{aligned}
$$

As $\tau>\sigma$, we have

$$
\lim _{m \rightarrow \infty} \min \left\{F_{2}\left(\mathcal{D}\left(\Omega^{2} u_{m}, \Omega^{2} u_{m-1}\right)\right), F_{1}\left(\mathcal{D}\left(\Omega^{3} u_{m}, \Omega^{3} u_{m-1}\right)\right)\right\}=-\infty
$$

Therefore, relation (44) implies that

$$
\lim _{m \rightarrow \infty} F_{2}\left(\mathcal{D}\left(\Omega^{2} u_{m-1}, \Omega^{2} u_{m}\right)\right)=-\infty
$$

or 


$$
\lim _{m \rightarrow \infty} F_{1}\left(\mathcal{D}\left(\Omega^{3} u_{m-1}, \Omega^{3} u_{m}\right)\right)=-\infty
$$

Using the analysis of the previous theorem, we will have

$$
\min \left\{F_{2}(\varepsilon), F_{1}(\varepsilon)\right) \geq F(\varepsilon)+\tau .
$$

That yields either $F_{2}(\varepsilon) \geq F(\varepsilon)+\tau$ or $F_{1}(\varepsilon) \geq F(\varepsilon)+\tau$. Now, with the usage of condition (B), we can write $F_{2}(\varepsilon) \geq F(\varepsilon)+\tau$ or $F_{2}(\varepsilon)>F(\varepsilon)+\sigma$, which implies, $F_{2}(\varepsilon)>F_{2}(\varepsilon)$. It is a contradiction. Similarly, $F_{1}(\varepsilon) \geq F(\varepsilon)+\tau$. Also, it yields a contradiction.

Thus, $\left\{u_{m}\right\}_{m=1}^{\infty}$ is a Cauchy sequence. The completeness of $(X, \mathcal{D})$ proves that $\left\{u_{m}\right\}_{m=1}^{\infty}$ converges to some point $u^{*}$ in $X$. Now, the continuity of $\Omega$ implies

$$
\mathcal{D}(\Omega u, u)=\lim _{m \rightarrow \infty} \mathcal{D}\left(\Omega u_{m}, u_{m}\right)=\lim _{m \rightarrow \infty} \mathcal{D}\left(\Omega u_{m+1}, u_{m}\right)=\mathcal{D}\left(u^{*}, u^{*}\right)=0 .
$$

Therefore, $\Omega$ has a fixed point $u^{*}$ in $X$, that is, $\Omega u^{*}=u^{*}$. So that $u^{*}=T u^{*}$. Now, for uniqueness, let us suppose that $T$ has more than one fixed point. That is, there exist two distinct $u, v \in X$ such that $T u=u \neq v=T v$. Therefore, $\mathcal{D}(u, v)=\mathcal{D}(T u, T v)=\mathcal{D}\left(T^{2} u, T^{2} v\right)>0$ with $\min \left\{F_{2}\left(\mathcal{D}\left(T^{2} u, T^{2} v\right)\right), F_{1}(\mathcal{D}(T u, T v))\right\} \geq F(\mathcal{D}(u, v))+\tau$ implies that either

$$
F_{1}(\mathcal{D}(u, v))=F_{1}(\mathcal{D}(T u, T v)) \geq F(\mathcal{D}(u, v))+\tau>F(\mathcal{D}(u, v))+\sigma>F_{1}(\mathcal{D}(u, v))
$$

or

$$
F_{2}(\mathcal{D}(u, v))=F_{2}\left(\mathcal{D}\left(T^{2} u, T^{2} v\right)\right) \geq F(\mathcal{D}(u, v))+\tau>F(\mathcal{D}(u, v))+\sigma>F_{2}(\mathcal{D}(u, v)) .
$$

Since both relations (45) and (46) are contradictions, the mapping $T$ has a unique fixed point.

\section{Applications to Caputo fractional differential equations}

As applications of our work, we will study the existence of solutions of Caputo fractional differential equations of the fractional order in $(1,2)$ with an integral boundary condition. The main condition in the problems studied in [1] (see Theorem 3.2 therein) and [2] (see Theorem 12 therein) is associated with sufficient small Lipschitz constant. We will use a less restrictive condition than the Lipschitz condition by applying our obtained fixed point theorem.

For $1<\mathfrak{r}<2$ and a Caputo fractional derivative ${ }_{\alpha}^{C} D_{t}^{\mathfrak{r}} x(t)=\frac{1}{\Gamma(2-\mathfrak{r})} \int_{\alpha}^{t}(t-s)^{1-\mathfrak{r}} x^{\prime \prime}(s) d s$, consider a nonlinear Caputo fractional differential equation

$$
{ }_{\alpha}^{C} D_{\mathfrak{t}}^{\mathfrak{r}}(x(\mathfrak{t}))=f(\mathfrak{t}, x(\mathfrak{t})) \quad \text { for } \mathfrak{t} \in(\alpha, \beta)
$$

with an integral boundary condition

$$
x(\alpha)=0, x(\beta)=\int_{\alpha}^{\lambda} x(s) d s \quad(\alpha<\lambda<\beta)
$$

where, for some $\lambda \in(\alpha, \beta), x(\lambda) \in \mathbb{R}$ and $\alpha, \beta$ are given real numbers such that $0 \leq \alpha<\beta$.

Let $\Omega=C([\alpha, \beta], \mathbb{R})$ be endowed with a norm $\|x\|_{[\alpha, \beta]}=\sup _{s \in[\alpha, \beta]}|x(s)|$.

For any $x, y \in \Omega$, we define $\mathcal{W}(x, y)=\|x-y\|_{[\alpha, \beta]}$. 
In order to assure the existence of solution of nonlinear Caputo fractional differential equation (47), we consider the following fractional differential equation:

$$
{ }_{\alpha}^{C} D_{t}^{\mathfrak{r}}(x(t)=g(t) \quad \text { for } t \in(\alpha, \beta) .
$$

Kilbas [40] proved that the following function

$$
\begin{aligned}
x(t)= & \frac{1}{\Gamma(\mathfrak{r})} \int_{\alpha}^{t}(t-s)^{\mathfrak{r}-1} g(s) d s \\
& +\frac{2(t-\alpha)}{\left((\lambda-\alpha)^{2}-2(\beta-\alpha)\right) \Gamma(\mathfrak{r})} \int_{\alpha}^{\beta}(\beta-s)^{\mathfrak{r}-1} g(s) d s \\
& -\frac{2(t-\alpha)}{\left((\lambda-\alpha)^{2}-2(\beta-\alpha)\right) \Gamma(\mathfrak{r})} \int_{\alpha}^{\lambda} \int_{\alpha}^{s}(s-\xi)^{\mathfrak{r}-1} g(\xi) d \xi d s
\end{aligned}
$$

represents the solution of boundary value problem (48) and (49) for $g \in \Omega$, based on the following presentation of the solution:

$$
x(t)=\frac{1}{\Gamma(\mathfrak{r})} \int_{\alpha}^{t}(t-s)^{\mathfrak{r}-1} g(s) d s-d_{1}-d_{2}(t-\alpha) .
$$

Next, we will define a mild solution of (47) and (48).

Definition 4.1 The function $x \in \Omega$ is a mild solution of boundary value problem (47) and (48) if it satisfies

$$
\begin{aligned}
x(t)= & \frac{1}{\Gamma(\mathfrak{r})} \int_{\alpha}^{t}(t-s)^{\mathfrak{r}-1} \mathcal{T}(s, x(s)) d s \\
& +\frac{2(t-\alpha)}{\left((\lambda-\alpha)^{2}-2(\beta-\alpha)\right) \Gamma(\mathfrak{r})} \int_{\alpha}^{\beta}(\beta-s)^{\mathfrak{r}-1} \mathcal{T}(s, x(s)) d s \\
& -\frac{2(t-\alpha)}{\left((\lambda-\alpha)^{2}-2(\beta-\alpha) \Gamma(\mathfrak{r})\right.} \int_{\alpha}^{\lambda} \int_{\alpha}^{s}(s-\xi)^{\mathfrak{r}-1} \mathcal{T}(\xi, x(\xi)) d \xi d s, \quad t \in[\alpha, \beta] .
\end{aligned}
$$

For any function $u \in \Omega$, we define a surjective mapping $\Upsilon: \Omega \rightarrow \Omega$ by

$$
\begin{aligned}
\Upsilon(u)(t)= & \frac{1}{\Gamma(\mathfrak{r})} \int_{\alpha}^{t}(t-s)^{\mathfrak{r}-1} \mathcal{T}(s, u(s)) d s \\
& +\frac{2(t-\alpha)}{\left((\lambda-\alpha)^{2}-2(\beta-\alpha) \Gamma(\mathfrak{r})\right.} \int_{\alpha}^{\beta}(\beta-s)^{\mathfrak{r}-1} \mathcal{T}(s, u(s)) d s \\
& -\frac{2(t-\alpha)}{\left((\lambda-\alpha)^{2}-2(\beta-\alpha)\right) \Gamma(\mathfrak{r})} \int_{\alpha}^{\lambda} \int_{\alpha}^{s}(s-\xi)^{\mathfrak{r}-1} \mathcal{T}(\xi, u(\xi)) d \xi d s
\end{aligned}
$$

for $t \in[\alpha, \beta]$.

Now, we establish the existence result as follows. 
(i) There exist a constant $K>0$ with

$$
\frac{K(\beta-\alpha) \mathfrak{r}}{\Gamma(1+\mathfrak{r})}\left(1+\frac{2 K(\beta-\alpha)}{\left(2(\beta-\alpha)-(\lambda-\alpha)^{2}\right)}\left(1+\frac{\lambda-\alpha}{1+\mathfrak{r}}\right)\right) \in(0, \infty)
$$

and a function $\mathcal{T} \in C([\alpha, \beta] \times \mathbb{R}, \mathbb{R})$ such that

$$
|\mathcal{T}(t, x)-\mathcal{T}(t, y)| \leq K|x-y|^{r}, \quad x, y \in \mathbb{R}, t \in[\alpha, \beta]
$$

where $r \in(0,1]$;

(ii) There exists a function $x_{0} \in \Omega$ such that $\mathcal{W}\left(x_{0}, \Upsilon\left(x_{0}\right)\right)>0$, where the operator $\Upsilon$ is defined by (52);

(iii) For any two functions $x, y \in \Omega$ such that $\mathcal{W}(x, y)>0$, the inequality $\mathcal{W}(\Upsilon(x), \Upsilon(y))>0$ holds;

Then boundary value problem (47) and (48) has a mild solution.

Proof Note that any fixed point of the mapping $\Upsilon$ is a mild solution of boundary value problem (47) and (48). Now, let $x, y \in \Omega$ be such that $\mathcal{W}(x, y)>0$. By condition (i), we obtain

$$
\begin{aligned}
& |\Upsilon(x)(t)-\Upsilon(y)(t)| \\
& \leq \frac{1}{\Gamma(\mathfrak{r})} \int_{\alpha}^{t}(t-s)^{\mathfrak{r}-1}|\mathcal{T}(s, x(s))-\mathcal{T}(s, y(s))| \mathcal{W} s \\
& +\frac{2(t-\alpha)}{\left(2(\beta-\alpha)-(\lambda-\alpha)^{2}\right) \Gamma(\mathfrak{r})} \int_{\alpha}^{\beta}(1-s)^{\mathfrak{r}-1}|\mathcal{T}(s, x(s))-\mathcal{T}(s, y(s))| \mathcal{W} s \\
& +\frac{2(t-\alpha)}{\left(2(\beta-\alpha)-(\lambda-\alpha)^{2}\right) \Gamma(\mathfrak{r})} \int_{\alpha}^{\lambda}\left(\int_{\alpha}^{s}(s-t)^{\mathfrak{r}-1}|\mathcal{T}(t, x(t))-\mathcal{T}(t, y(t))| \mathcal{W} t\right) \mathcal{W} s \\
& \leq \frac{K}{\Gamma(\mathfrak{r})} \int_{\alpha}^{t}(t-s)^{\mathfrak{r}-1}|x(s)-y(s)|^{r} d s \\
& +\frac{2 K(t-\alpha)}{\left(2(\beta-\alpha)-(\lambda-\alpha)^{2}\right) \Gamma(\mathfrak{r})} \int_{\alpha}^{\beta}(\beta-s)^{\mathfrak{r}-1}|x(s)-y(s)|^{r} d s \\
& +\frac{2 K(t-\alpha)}{\left(2(\beta-\alpha)-(\lambda-\alpha)^{2}\right) \Gamma(\mathfrak{r})} \int_{\alpha}^{\lambda}\left(\int_{\alpha}^{s}(s-\xi)^{\mathfrak{r}-1}|x(\xi)-y(\xi)|^{r} d \xi\right) d s \\
& \leq\left(\frac{K(t-\alpha)^{\mathfrak{r}}}{\mathfrak{r} \Gamma(\mathfrak{r})}+\frac{2 K(t-\alpha)}{\left(2(\beta-\alpha)-(\lambda-\alpha)^{2}\right) \Gamma(\mathfrak{r})}\left(\frac{(\beta-\alpha)^{\mathfrak{r}}}{\mathfrak{r}}+\frac{(\lambda-\alpha)^{1+\mathfrak{r}}}{\mathfrak{r}(1+\mathfrak{r})}\right)\right)\|x-y\|_{\infty}^{r} \\
& \leq \frac{K(\beta-\alpha)^{\mathfrak{r}}}{\Gamma(1+\mathfrak{r})}\left(1+\frac{2 K(\beta-\alpha)}{\left(2(\beta-\alpha)-(\lambda-\alpha)^{2}\right)}\left(1+\frac{\lambda-\alpha}{1+\mathfrak{r}}\right)\right)\|x-y\|_{\infty}^{r} \\
& =\Lambda\|x-y\|_{\infty}^{r}, \quad t \in[\alpha, \beta],
\end{aligned}
$$

where

$$
\Lambda=\frac{K(\beta-\alpha)^{\mathfrak{r}}}{\Gamma(1+\mathfrak{r})}\left(1+\frac{2 K(\beta-\alpha)}{\left(2(\beta-\alpha)-(\lambda-\alpha)^{2}\right)}\left(1+\frac{\lambda-\alpha}{1+\mathfrak{r}}\right)\right) \in(0, \infty)
$$


Therefore,

$$
\|\Upsilon(x)-\Upsilon(y)\|_{\infty} \leq \Lambda\|x-y\|_{\infty}^{r} .
$$

The conditions of the theorem imply that $\Upsilon(x) \neq \Upsilon(y)$ if and only if $x \neq y$. Therefore, $\Upsilon$ : $\Omega \rightarrow \Omega$ is a bijective mapping. There exists $\chi: \Omega \rightarrow \Omega$ such that $\Upsilon \chi=I=\chi \Upsilon$ ( $I$ the identity mapping).

Next, we suppose that $f=\Upsilon(x)$ and $h=\Upsilon(y)$ such that $\chi(f)=x$ and $\chi(h)=y$.

So that

$$
\|f-h\|_{\infty} \leq \Lambda\|\chi(f)-\chi(h)\|_{\infty}^{r} .
$$

That implies that

$$
\|\chi(f)-\chi(h)\|_{\infty} \geq\left(\frac{1}{\Lambda}\right)^{1 / r}\|f-h\|_{\infty}^{1 / r} .
$$

Take $\kappa>\Lambda$, so one writes

$$
(\kappa)^{1 / r}\|\chi(f)-\chi(h)\|_{\infty} \geq\left(\frac{\kappa}{\Lambda}\right)^{1 / r}\|f-h\|_{\infty}^{1 / r}
$$

Moreover, (55) further implies that

$$
\begin{aligned}
\left\|\chi^{2}(f)-\chi^{2}(h)\right\|_{\infty} & \geq\left(\frac{1}{\Lambda}\right)^{1 / r}\|\chi(f)-\chi(h)\|_{\infty}^{1 / r} \\
& \geq\left(\frac{1}{\Lambda}\right)^{\left(\frac{1}{r}+\frac{1}{r^{2}}\right)}\|f-h\|_{\infty}^{1 / r^{2}} .
\end{aligned}
$$

So that

$$
\kappa^{\left(\frac{1}{r}+\frac{1}{r^{2}}\right)}\left\|\chi^{2}(f)-\chi^{2}(h)\right\|_{\infty} \geq\left(\frac{\kappa}{\Lambda}\right)^{\left(\frac{1}{r}+\frac{1}{r^{2}}\right)}\|f-h\|_{\infty}^{1 / r^{2}} .
$$

Relation (56) yields that

$$
\ln (\kappa)^{1 / r}\|\chi(f)-\chi(h)\|_{\infty} \geq \frac{1}{r} \ln \left(\frac{\kappa}{\Lambda}\right)+\ln \|f-h\|_{\infty}^{1 / r} .
$$

Likewise, relation (56) can be written as

$$
\begin{aligned}
\ln \kappa^{\left(1+\frac{1}{r}\right)}\left\|\chi^{2}(f)-\chi^{2}(h)\right\|_{\infty}^{r} & \geq \ln \left(\frac{\kappa}{\Lambda}\right)+\frac{1}{r} \ln \left(\frac{\kappa}{\Lambda}\right)+\ln \|f-h\|_{\infty}^{1 / r} \\
& >\frac{1}{r} \ln \left(\frac{\kappa}{\Lambda}\right)+\ln \|f-h\|_{\infty}^{1 / r} .
\end{aligned}
$$

Let $F_{2}(z)=\ln \kappa^{\left(1+\frac{1}{r}\right)}(z)^{r}, F_{1}(z)=\ln (\kappa)^{1 / r}(z)$, and $F(z)=\ln (z)^{1 / r}$. It is easy to verify that $F, F_{1}, F_{2} \in \mathcal{F}$. 
Combining relations (57) and (59), we have

$$
\min \left\{F_{2}\left(\mathcal{W}\left(\chi^{2}(f), \chi^{2}(h)\right)\right), F_{1}(\mathcal{W}(\chi(f), \chi(h)))\right\} \geq \frac{1}{r} \ln \left(\frac{\kappa}{\Lambda}\right)+F(\mathcal{W}(f, h))
$$

Therefore, $\chi: \Omega \rightarrow \Omega$ is a triple $F$-expanding mapping, and the operator $\chi$ has a fixed point in $\Omega$. That is, there exists a function $x^{*} \in C([\alpha, \beta], \mathbb{R})$ such that $x^{*}=\chi\left(x^{*}\right)$. This implies that $\Omega\left(x^{*}\right)=x^{*}$. The function $x^{*}$ is a mild solution of boundary value problem (47) and (48).

Example 4.1 Consider the nonlinear Caputo fractional differential equation

$$
{ }_{2}^{C} D_{t}^{1.75}(x(t))=\frac{1}{\sqrt{t+14}} \arctan \left(\sqrt{|x(t)|}+e^{t} \cos t\right)+\sin t \quad \text { for } t \in(2,3)
$$

with the integral boundary conditions

$$
x(2)=0, \quad x(3)=\int_{0}^{2.5} x(s) d s .
$$

Here, $\mathfrak{r}=1.75$. Also,

$$
\mathcal{T}(t, x)=\frac{1}{\sqrt{t+14}} \arctan \left(\sqrt{|x|}+e^{t} \cos t\right)+\sin t
$$

and

$$
|\mathcal{T}(t, x)-\mathcal{T}(t, y)| \leq\left(\frac{\pi}{4}+1\right) \sqrt{|x-y|}
$$

where

$$
\begin{aligned}
\Lambda & =\frac{K(\beta-\alpha)^{\mathfrak{r}}}{\Gamma(1+\mathfrak{r})}\left(1+\frac{2 K(\beta-\alpha)}{\left(2(\beta-\alpha)-(\lambda-\alpha)^{2}\right)}\left(1+\frac{\lambda-\alpha}{1+\mathfrak{r}}\right)\right) \\
& =\frac{\left(\frac{\pi}{4}+1\right)}{\Gamma(2.75)}\left(1+\frac{2\left(\frac{\pi}{4}+1\right)}{1.75} \frac{3.25}{2.75}\right) \in(0, \infty) .
\end{aligned}
$$

Note that $\Lambda>1$. Therefore, Theorem 4.1 guarantees the existence of a solution of boundary value problem (60) and (61).

Remark 4.1 Note that boundary value problem (60) and (61) is also studied in [1] (see Example 5 therein) and [2] (see Example 3.3 therein). Based on the obtained fixed point theorems, we used the weaker conditions for the right-hand side part of the equation and found the existence of a fixed point for $K>1$ and $\Lambda>1$.

\section{An application to integral equations}

As another application of our work, we will consider an engineering problem in which the transformed mathematical model of a problem representing activation of spring affected 
by an external force is a boundary value problem for a second-order differential equation. That is,

$$
\ddot{u}+\frac{c}{m} \dot{u}=H(\mathfrak{w}, u(\mathfrak{w})) ; \quad u(0)=0, \dot{u}(0)=a,
$$

where $H:[0, I] \times \mathbb{R}^{+} \rightarrow \mathbb{R}^{+}$is a continuous function and $I>0$.

Conversion of the given problem into the following integral equation is well known:

$$
u(\mathfrak{r})=\int_{0}^{\mathfrak{r}} H(\mathfrak{w}, u(\mathfrak{w})) G(\mathfrak{r}, \mathfrak{w}), \quad \mathfrak{r} \in[0, I]
$$

Define the Green function $G(\mathfrak{r}, \mathfrak{w})$ as

$$
G(\mathfrak{r}, \mathfrak{w})= \begin{cases}(\mathfrak{r}+\mathfrak{w}) e^{\tau(\mathfrak{r}-\mathfrak{w})} & \text { if } 0 \leq \mathfrak{w} \leq \mathfrak{r} \leq I \\ 0 & \text { if } 0 \leq \mathfrak{r} \leq \mathfrak{w} \leq I\end{cases}
$$

with a constant $\tau=\tau(c, m)>0$.

Let $X$ be the set of all continuous functions from $[0, I]$ into $\mathbb{R}^{+}$. For any arbitrary $U \in X$, define

$$
\|U\|_{\tau}=\sup _{\mathfrak{r} \in[0, I]}\left\{|U(\mathfrak{r})| e^{-2 \tau \mathfrak{r}}\right\} .
$$

Define $\mathcal{D}: X \times X \rightarrow[0, \infty)$ by

$$
\mathcal{D}(x, y)=\max \left\{\|x\|_{\tau},\|y\|_{\tau}\right\}
$$

for all $x, y \in X$.

Now, in order to find the existence of a solution to the integral equation, we consider a function $g: X \rightarrow X$ defined by

$$
g(u(\mathfrak{r}))=\int_{0}^{\mathfrak{r}} H(\mathfrak{w}, u(\mathfrak{w})) G(\mathfrak{r}, \mathfrak{w})
$$

for all $\mathrm{u} \in X$ and $\mathfrak{r} \in[0, I]$.

We will prove that there exists some $v \in X$ such that $g(v(\mathfrak{r}))=v(\mathfrak{r})$. That is, the fixed point of the $F$-expanding mapping is a solution of integral equation (62).

Theorem 5.1 The nonlinear integral equation (58) has a solution if the following conditions hold:

(a) $H(\mathfrak{w}, u(\mathfrak{w}))$ is an increasing function in its second variable;

(b) $H(\mathfrak{w}, u) \geq k \tau^{2} e^{\tau} u, k>0$ such thatr $\tau \geq 1+\frac{1}{2 e^{2}}$, where $\tau>0, \mathfrak{r}, \mathfrak{w} \in[0, I]$, and $u \in \mathbb{R}^{+}$;

(c) $g: X \rightarrow X$ is a surjective continuous mapping.

Proof For all $v \in X$, we have

$$
|g(v(\mathfrak{w}))|=g(v(\mathfrak{w}))=\int_{0}^{\mathfrak{r}} H(\mathfrak{w}, v(\mathfrak{w})) G(\mathfrak{r}, \mathfrak{w}) d \mathfrak{w} .
$$


Now, using conditions (a) and (b), we can write

$$
\begin{aligned}
|g(v(\mathfrak{w}))| & \geq k \int_{0}^{\mathfrak{r}} \tau^{2} e^{\tau}|v(\mathfrak{w})| G(\mathfrak{r}, \mathfrak{w}) d \mathfrak{w} \\
& =k \int_{0}^{\mathfrak{r}} \tau^{2} e^{\tau}|v(\mathfrak{w})|(\mathfrak{r}+\mathfrak{w}) e^{\tau(\mathfrak{r}-\mathfrak{w})} d \mathfrak{w} \\
& =k \int_{0}^{\mathfrak{r}} \tau^{2} e^{\tau} e^{2 \tau \mathfrak{w}} e^{-2 \tau \mathfrak{w}}|v(\mathfrak{w})|(\mathfrak{r}+\mathfrak{w}) e^{\tau(\mathfrak{r}-\mathfrak{w})} d \mathfrak{w} \\
& =k \int_{0}^{\mathfrak{r}} \tau^{2} e^{\tau} e^{2 \tau \mathfrak{w}}\|v\|_{\tau}(\mathfrak{r}+\mathfrak{w}) e^{\tau(\mathfrak{r}-\mathfrak{w})} d \mathfrak{w} \\
& =k \tau^{2} e^{\tau} e^{\mathfrak{r} \tau}\|v\|_{\tau} \int_{0}^{\mathfrak{r}} e^{2 \tau \mathfrak{w}}(\mathfrak{r}+\mathfrak{w}) e^{\tau \mathfrak{w}} d \mathfrak{w} \\
& =k \tau^{2} e^{\mathfrak{r} \tau+\tau}\|v\|_{\tau}\left(2 \frac{\mathfrak{r} e^{\tau \mathfrak{r}}}{\tau}-\frac{\mathfrak{r}}{\tau}-\frac{e^{\tau \mathfrak{r}}}{\tau^{2}}+\frac{1}{\tau^{2}}\right) \\
& =k e^{\mathfrak{r} \tau+\tau}\|v\|_{\tau}\left(2 \mathfrak{r} \tau e^{\tau \mathfrak{r}}-\mathfrak{r} \tau-e^{\tau \mathfrak{r}}+1\right) \\
& =k e^{2 \tau \mathfrak{r}} e^{\tau}\|v\|_{\tau}\left(2 \mathfrak{r} \tau-\mathfrak{r} \tau e^{-\tau \mathfrak{r}}-1+e^{-\tau \mathfrak{r}}\right) .
\end{aligned}
$$

Therefore,

$$
e^{-2 \tau \mathfrak{r}}|g(v(\mathfrak{w}))| \geq k e^{\tau}\|v\|_{\tau}\left(2 t \tau-1+(1-\mathfrak{r} \tau) e^{-\tau \mathfrak{r}}\right) .
$$

Using condition (b), one gets

$$
2 \mathfrak{r} \tau-1+(1-\mathfrak{r} \tau) e^{-\tau \mathfrak{r}} \geq 1 .
$$

That is,

$$
\|g(v(\mathfrak{w}))\|_{\tau} \geq k e^{\tau}\|v\|_{\tau}
$$

Likewise, for $w \in X$, we can find that

$$
\| g(w))\left\|_{\tau} \geq k e^{\tau}\right\| w \|_{\tau}
$$

Since

$$
\max \left\{\|g v\|_{\tau},\|g w\|_{\tau}\right\} \geq \max \left\{k e^{\tau}\|v\|_{\tau}, k e^{\tau}\|w\|_{\tau}\right\},
$$

we have

$$
\mathcal{D}(g v, g w) \geq k e^{\tau} \max \left\{\|v\|_{\tau},\|w\|_{\tau}\right\} .
$$

Equivalently,

$$
\mathcal{D}(g v, g w) \geq k e^{\tau} \mathcal{D}(v, w) .
$$


Therefore,

$$
\ln \left(\frac{1}{k} \mathcal{D}(g v, g w)\right) \geq \ln (\mathcal{D}(v, w))+\tau .
$$

Now, relation (64) also implies that

$$
\mathcal{D}\left(g^{2} v, g^{2} w\right) \geq k^{2} e^{2 \tau} \mathcal{D}(v, w) .
$$

That is,

$$
\frac{1}{k^{2}} \mathcal{D}\left(g^{2} v, g^{2} w\right) \geq e^{2 \tau} \mathcal{D}(v, w)
$$

Thus,

$$
\ln \left(\frac{1}{k^{2}} \mathcal{D}\left(g^{2} v, g^{2} w\right)\right) \geq \ln (\mathcal{D}(v, w))+2 \tau .
$$

We deduce

$$
\ln \left(\frac{1}{k^{2}} \mathcal{D}\left(g^{2} v, g^{2} w\right)\right)>\ln (\mathcal{D}(v, w))+\tau .
$$

Define $F_{1}(x)=\ln \frac{x}{k}, F_{2}(x)=\ln \frac{x}{k^{2}}$, and $F(x)=\ln x$. So that inequalities (65) and (66) take the form

$$
F_{1}(\mathcal{D}(T v, T w)) \geq F(\mathcal{D}(v, w))+\tau \quad \text { and } \quad \mathrm{F}_{2}\left(\mathcal{D}\left(T^{2} v, T^{2} w\right)\right)>F(\mathcal{D}(v, w))+\tau
$$

Both of the above inequalities can be written as

$$
\min \left\{F_{1}(\mathcal{D}(T v, T w)), \mathrm{F}_{2}\left(\mathcal{D}\left(T^{2} v, T^{2} w\right)\right)\right\} \geq F(\mathcal{D}(v, w))+\tau
$$

Therefore, the fixed point of the above triple $F$-contraction is the solution of problem (62).

\section{Conclusion}

In this article, we generalized $F$-expanding mappings by using multiple $F$ functions and certain conditions on the mapping. These conditions allow us to deal with the class of mappings whose iterates expand in general, but some of their iterates may contract as well. Moreover, with the usage of multiple $F$ functions, we presented an idea that allows to use weaker conditions for several fractional type differential equations. The new generalizations of $F$-expanding mappings and the corresponding results will break open new grounds for the researchers working in the field as they will be able to find the existence of solutions of an extensive range of differential equations. 
Availability of data and materials

Data sharing is not applicable to this article as no data sets were generated or analysed during the current study.

\section{Competing interests}

The authors declare that they have no competing interests.

\section{Authors' contributions}

All the authors have equally contributed to the final manuscript. All authors read and approved the final manuscript.

\section{Author details}

'National University of Computer and Emerging Sciences, Lahore, 54700, Pakistan. ${ }^{2}$ Department of Mathematics, University of Management and Technology, Lahore, 54700, Pakistan. ${ }^{3}$ Institut Supérieur d'Informatique et des Techniques de Communication, Université de Sousse, Sousse, Tunisia. ${ }^{4}$ China Medical University Hospital, China Medical University, Taichung, 40402, Taiwan. ${ }^{5}$ Department of Mathematics and Applied Mathematics, Sefako Makgatho Health Sciences University, Ga-Rankuwa, South Africa. ${ }^{6}$ Department of Mathematics, College of science and Arts, Qassim University, Buraydah, Saudi Arabia.

\section{Publisher's Note}

Springer Nature remains neutral with regard to jurisdictional claims in published maps and institutional affiliations.

Received: 25 February 2021 Accepted: 8 July 2021 Published online: 31 July 2021

\section{References}

1. Mehmood, N., Ahmad, N.: Existence results for fractional order boundary value problem with nonlocal non-separated type multi-point integral boundary conditions. AIMS Math. 5, 385-398 (2019)

2. Hanadi, Z., Hoda, A., Fouad, S.H., Jamshaid, A.: Generalized fixed point results with application to nonlinear fractional differential equations. Mathematics 8, 1168 (2020)

3. Wardowski, D.: Fixed point theory of a new type of contractive mappings in complete metric spaces. Fixed Point Theory Appl. 2016, 94 (2016)

4. Karapinar, E., Agarwal, R.P., Aydi, H.: Interpolative Reich-Rus-Ciric type contractions on partial metric spaces. Mathematics 6(11), 256 (2018)

5. Alqahtani, B., Fulga, A., Jarad, F., Karapinar, E.: Nonlinear F-contractions on b-metric spaces and differential equations in the frame of fractional derivatives with Mittag-Leffler kernel. Chaos Solitons Fractals 128, 349-354 (2019)

6. Aydi, H., Karapinar, E., Shatanawi, W.: Coupled fixed point results for $(\psi, \varphi)$-weakly contractive condition in ordered partial metric spaces. Comput. Math. Appl. 62, 4449-4460 (2011)

7. Aydi, H., Mlaiki, N., Souayah, N., Abdeljawad, T.: Controlled metric type spaces and the related contraction principle. Mathematics 6, 194 (2018)

8. Secelean, N.A.: Weak F-contractions and some fixed point results. Bull. Iran. Math. Soc. 42(3), 779-798 (2016)

9. Piri, H., Kumam, P.: Fixed point theorems for generalized F-Suzuki-contraction mappings in complete $b$-metric spaces. Fixed Point Theory Appl. 2016, 90 (2016)

10. Piri, H., Kumam, P.: Some fixed point theorems concerning F-contraction in complete metric spaces. Fixed Point Theory Appl. 2014, 210 (2014)

11. Mınak, G., Helvacı, A., Altun, I.: Ćirić type generalized F-contractions on complete metric spaces and fixed point results. Filomat 28(6), 1143-1151 (2014)

12. Vetro, F.: F-contractions of Hardy-Rogers type and application to multistage decision processes. Nonlinear Anal., Model. Control 21(4), 531-546 (2016)

13. Secelean, N.A., Wardowski, D.: \& F-contractions: not necessarily nonexpansive Picard operators. Results Math. 70, 415-431 (2016)

14. Lukács, A., Kajántó, S.: Fixed point results for various type F-contractions in complete b-metric spaces. Fixed Point Theory 19(1), 321-334 (2018)

15. Ameer, E., Aydi, H., Arshad, M., De la Sen, M.: Hybrid Ćirić type graphic $(\Upsilon, \Lambda)$-contraction mappings with applications to electric circuit and fractional differential equations. Symmetry 12(3), 467 (2020)

16. Parvaneh, V., Haddadi, M.R., Aydi, H.: On best proximity point results for some type of mappings. J. Funct. Spaces 2020, Article ID 6298138 (2020)

17. Patle, P., Patel, D., Aydi, H., Radenović, S.: On $H^{+}$-type multivalued contractions and applications in symmetric and probabilistic spaces. Mathematics 7(2), 144 (2019)

18. Ameer, E., Aydi, H., Arshad, M., Alsamir, H., Noorani, M.S.: Hybrid multivalued type contraction mappings in $\alpha_{K}$-complete partial b-metric spaces and applications. Symmetry 11(1), 86 (2019)

19. Gilić, E., Dolićanin-Dekić, D., Mitrović, Z.D., Pučić, D., Aydi, H.: On some recent results concerning F-Suzuki-contractions in b-metric spaces. Mathematics 8, 940 (2020)

20. Abbas, M., Berzig, M., Nazir, T., Karapinar, E.: Iterative approximation of fixed points for presic type F-contraction operators. UPB Sci. Bull., Ser. A 78(2), 147-160 (2016)

21. Hammad, H.A., Aydi, H., Mlaiki, N.: Contributions of the fixed point technique to solve the 2D Volterra integral equations, Riemann-Liouville fractional integrals, and Atangana-Baleanu integral operators. Adv. Differ. Equ. 2021, 97 (2021)

22. Adigüzel, R.S., Aksoy, U., Karapinar, E., Erhan, I.M.: On the solution of a boundary value problem associated with a fractional differential equation. Math. Methods Appl. Sci. (2020)

23. Aydi, H., Karapinar, E., Roldan Lopez de Hierro, A.F.: w-interpolative Ciric-Reich-Rus type contractions. Mathematics 7(1), 57 (2019)

24. Aydi, H., Chen, C.M., Karapinar, E.: Interpolative Ciric-Reich-Rus type contractions via the Branciari distance. Mathematics 7(1), 84 (2019) 
25. Ali, M.U., Aydi, H., Alansari, M.: New generalizations of set valued interpolative Hardy- Rogers type contractions in b-metric spaces. J. Funct. Spaces 2021, Article ID 6641342 (2021)

26. Gornicki, J.: Fixed point theorems for F-expanding mappings. Fixed Point Theory Appl. 2017, 9 (2017)

27. Budhia, L.B., Kumam, P., Martinez-Moreno, J., Gopal, D.: Extensions of almost- $F$ and $F$-Suzuki contractions with graph and some applications to fractional calculus. Fixed Point Theory Appl. 2016, 2 (2016)

28. Karapinar, E., Czerwik, C., Aydi, H.: $(\alpha, \psi)$-Meir-Keeler contraction mappings in generalized b-metric spaces. J. Funct. Spaces 2018, Article ID 3264620 (2018)

29. Gopal, D., Abbas, M., Patel, D.K., Vetro, C.: Fixed points of $a$-type F-contractive mappings with an application to nonlinear fractional differential equation. Acta Math. Sci. 36, 957-970 (2016)

30. Afshari, H., Kalantari, S., Karapınar, E.: Solution of fractional differential equations via coupled fixed point. Electron. J. Differ. Equ. 286(2015), 1 (2015)

31. Javed, K., Aydi, H., Uddin, F., Arshad, M.: On orthogonal partial b-metric spaces with an application. J. Math. 2021, Article ID 6692063 (2021)

32. Ahmad, B., Alsaedi, A., Alsharif, A.: Existence results for fractional-order differential equations with nonlocal multi-point-strip conditions involving Caputo derivative. Adv. Differ. Equ. 2015, 348 (2015)

33. Garrappa, R.: Numerical solution of fractional differential equations: a survey and a software tutorial. Mathematics 2018(6), 16 (2016)

34. Karapinar, E., Alqahtani, O., Aydi, H.: On interpolative Hardy-Rogers type contractions. Symmetry 11(1), 8 (2019)

35. Aydi, H., Jeli, M., Samet, B.: On positive solutions for a fractional thermostat model with a convex -concave source term via $\psi$-Caputo fractional derivative. Mediterr. J. Math. 17(1), 16 (2020)

36. Hammad, H.A., Aydi, H., Gaba, Y.U.: Exciting fixed point results on a novel space with supportive applications. J. Funct. Spaces 2021, Article ID 6613774 (2021)

37. Marasi, H.R., Aydi, H.: Existence and uniqueness results for two-term nonlinear fractional differential equations via a fixed point technique. J. Math. 2021, Article ID 6670176 (2021)

38. Karapınar, E., Fulga, A., Agarwal, R.P.: A survey: F-contractions with related fixed point results. J. Fixed Point Theory Appl. 13(22), 69 (2020)

39. Wardowski, D., Dung, N.V.: Fixed points of F-weak contractions on complete metric spaces. Demonstr. Math. 1, 146-155 (2014)

40. Kilbas, A.A., Srivastava, H.M., Trujillo, J.J.: Theory and Applications of Fractional Differential Equations. Elsevier, Amsterdam (2006)

\section{Submit your manuscript to a SpringerOpen ${ }^{\circ}$ journal and benefit from:}

- Convenient online submission

- Rigorous peer review

- Open access: articles freely available online

- High visibility within the field

Retaining the copyright to your article

Submit your next manuscript at $\boldsymbol{~ s p r i n g e r o p e n . c o m ~}$ 